\title{
A new predictor-corrector approach for the numerical integration of coupled electromechanical equations
}

\author{
E. Tripodi*, A. Musolino, R. Rizzo, M. Raugi \\ DESTEC, Department of Energy and Systems Engineering, University of Pisa, Largo Lucio Lazzarino, 56122, Italy
}

\section{SUMMARY}

In this paper a new approach for the numerical solution of coupled electromechanical problems is presented. The structure of the considered problem consists of the low frequency integral formulation of the Maxwells equations coupled with Newton-Euler rigid-body dynamic equations. Two different integration schemes based on the predictor-corrector approach are presented and discussed. In the first method the electrical equation is integrated with an implicit Single Step Time Marching Algorithm (SSTMA) while the mechanical dynamics is studied by a predictor-corrector scheme. The predictor uses the Forward Euler method while the corrector is based on the Trapezoidal Rule. The second method is based in the use of two interleaved predictor-corrector schemes: one for the electrical equations, the other for the mechanical ones. Both the presented methods have been validated by comparison with experimental data (when available) and with results obtained by other numerical formulations; in problems characterized by low speeds, both schemes produce accurate results, with similar computation times. When high speeds are involved, the first scheme needs shorter time steps (i.e. longer computation times) in order to achieve the same accuracy of the second one. A brief discussion on extending the algorithm for simulating deformable bodies is also presented. An example of application to a 2 degrees of freedom levitating device based on Permanent Magnets is finally reported.

Received ...

KEY WORDS: Electromechanical problem, computational electromagnetics, magnetic levitation.

\section{INTRODUCTION}

The numerical solution of coupled electromagnetic and mechanical problems is an open issue in the electromagnetic community. The analysis and design of all the electro-mechanical devices rely on the availability of robust and efficient numerical tools. In applications characterized by high speeds (such as ElectroMagnetic Launchers (EML), Magnetic Levitation (MAGLEV) systems, Permanent Magnet Bearings), assuming constant coefficients of the electrical equations during the time step adopted in the integration may be not valid, unless using very short time steps. This requires a strong coupling between electrical and mechanical equations [1-9].

The nowadays availability of NdFeB Permanent Magnets (PMs) has raised a lot of interest on MAGLEV transportation and on the development of magnetic bearings [10]. In this context, the main problem to deal with is the intrinsic mechanical instability of these configurations (EarnshawBraunbeck theorem) [11,12]. Consequently, an interesting challenge concerns the development of control systems which are able to dynamically stabilize the devices. Obtaining an accurate solution of the coupled electromechanical problem which is governed by unstable poles is very important since the accurate knowledge of the open loop evolution is essential for the design of the control system. Several papers [13-19] highlight the actual need of accurate choices of the geometry and configurations, also in the early design process of MAGLEV devices.

$\overline{\dagger E-m a i l: ~ e r n e s t o . t r i p o d i @ g m a i l . c o m ~}$ 
The low frequency formulation of the Maxwell equations and the hypothesis of rigid body are usually adopted in this context (see Appendix A).

In this paper we refer to the six Degrees of Freedom (DoF) coupled electromechanical problem. The numerical approach poses a number of critical issues. One of these is the choice of the formulation for the magnetic diffusion equations. Differential and integral formulations are at the basis of the most popular computational approaches: the Finite Element Method (FEM) and the Method of Moments (MoM) and their respective variations. FEM analysis is widely used since it allows modeling devices with complex shapes and characterized by nonlinear materials. The application of FEM to systems with moving conductors presents some difficulties due to the relative motion of the bodies and their own meshes. Despite the availability of commercial codes that are able to solve this problem [20-22] the search for robust and efficient methods is still open.

Alternative approaches, mostly based on integral formulations as the one described in this paper, have a number of characteristics that make them well suited for the analysis of electromechanical devices [23-25]. In particular integral formulations require the discretization of the active regions only and the problem of coupling meshes with different speed is absent. Integral formulations automatically satisfy the far-field boundary conditions and coarse discretizations (with respect to those typically used in FEM) are able to provide good accuracy. Furthermore integral formulations can be efficiently parallelized [26]. Most of MoM based approaches can be reformulated in terms of equivalent networks whose coupling with external lumped circuits is straightforward. This represents an important feature since the stabilization systems are substantially constituted by electromagnets whose currents are provided by the electronic circuits on the basis of control strategies exploiting real-time evaluated parameters.

A simulation environment which allows evaluating the behavior of the system to be stabilized together with the control devices and their drivers is a valuable aid to the design activities. Once all the currents (equivalent magnetization currents, induced currents on massive conductor and those feeding the control devices) are known, it is possible to evaluate the forces and the torques acting on the system and update velocity and position integrating the motion equations. This will affect the elements of the matrices describing the electromagnetic behavior. The tight coupling of the two problems is evident as well as the intrinsic nonlinearity.

A simplified integration scheme consists in updating the MoM matrices only when the relative displacements between the subparts of the system is greater than a fixed quantity. Increasing speeds require shorter steps and the evaluation of the MoM matrices at every step with consequent long computation times. The choice of an accurate and efficient integration algorithm is further complicated by the stiffness of the problem; i.e. the electrical and mechanical equations may have different time constants. Moreover, coupling between the equations makes difficult the use of explicit schemes because the driving terms (force and torque in mechanical equations and motion induced voltages in the electrical ones) are not known. Forces and torques on the mechanical equations depend on currents which have not been evaluated, and similarly the motion induced voltage (that appears on the electrical equations) depend on the velocity which is know only after the mechanical equations are integrated.

Prediction-correction coupled schemes are proposed in this paper to circumvent this problem. More specifically, two algorithms will be discussed: the first one weakens the coupling of the electrical and the mechanical equations and maintains the presence of the two dynamics each governed by its time constants. The second preserves the tight coupling and performs simultaneous integration of the electric and mechanical dynamics by using an integration step that can be chosen longer (at least twice with the same accuracy) than the one adopted for the integration of the mechanical dynamic in the first algorithm.

Throughout the paper we consider high speed devices those in which the rate of change of the electrical parameters due to the motion is comparable with the rate of change of the electrical quantities. For this kind of devices the application of the weak coupling would require very short time step in order to make reasonable the approximation of considering constant the electrical parameters during the time step. The use of the second scheme allows using longer time steps since the electrical parameters are assumed to vary linearly during the time step. The paper is organized 
as follows: Sec. 2 introduces and discusses the two algorithms, including a brief final discussion on deformable bodies. Sec. 3 describes the validation of both the procedures by comparison with experimental and numerical results, while Sec. 4 shows the results obtained simulating a particular 2 DoF application example. Finally, in the Appendix A, a more extended description of the coupled problem and the integral formulation are reported for the sake of clarity.

\section{PROBLEM FORMULATION AND TIME INTEGRATION SCHEMES}

\subsection{Problem formulation and time integration schemes}

The details of the adopted formulation are reported in [26-29] and for the sake of clarity a brief description has been reported in Appendix A.

For the sake of simplicity let us consider a system composed of two rigid bodies: one is attached to the coordinate system and the other is free to move without constraints (six degrees of freedom). We assume that the elementary volumes are numbered such that the first $N_{f}$ elementary volumes constitute the fixed body while the subsequent $N_{m}$ elementary volumes belong to the moving body. Under the hypothesis of linear magnetizable materials the governing equations of the coupled problem can be written as :

$$
\begin{aligned}
& \mathbf{L}(\mathbf{C}(t)) \frac{d \mathbf{i}}{d t}+[\mathbf{R}(\mathbf{C}(t))+\mathbf{K}(\mathbf{C}(t), \dot{\mathbf{C}}(t))] \mathbf{i}=\mathbf{e}(t) \\
& \mathbf{F}(\mathbf{C}(t), \mathbf{i})=m \ddot{\mathbf{q}} \\
& \mathbf{T}(\mathbf{C}(t), \mathbf{i})=\mathbf{I}_{\theta \theta} \dot{\boldsymbol{\omega}}+\boldsymbol{\omega} \times \mathbf{I}_{\theta \theta} \boldsymbol{\omega}
\end{aligned}
$$

where $\mathbf{i}$ is the vector of the currents in the elementary volumes, and e represents the vector of the applied voltage generators. It is worth to note that $\mathbf{i}$ include the equivalent magnetization currents and that some of the currents may be imposed: e.g. the equivalent magnetization currents of the PM or the conduction currents from ideal current generators. All the coefficients matrices are function of $\mathbf{C}(t)$ which represents the system configuration at the instant $t$. $\mathbf{C}(t)$ is defined as the set of the positions and orientations of all the elementary volumes in which the device is discretized. For the $i$-th elementary volume:

$$
\mathbf{C}_{i}(t)=\left(x_{i}(t), y_{i}(t), z_{i}(t), \phi_{i}(t), \theta_{i}(t), \psi_{i}(t)\right)
$$

where $x_{i}, y_{i}, z_{i}$ are vectors built with the coordinates of the centers of gravity of the elementary volume, and $\phi_{i}, \theta_{i}, \psi_{i}$ are the Euler angles (with XYZ convention) defining the orientation of the body fixed coordinate system of the elementary volume w.r.t. the global one. We can then define $\mathbf{C}(t)$ as:

$$
\mathbf{C}(t)=\left[\mathbf{C}_{i}(t)\right] \quad i=1, \ldots, N_{f}+N_{m}
$$

$\mathbf{L}(\mathbf{C}(t))$ denotes the inductance matrix. As an example, in the unrealistic situation of having both the fixed and rigid body consisting of just one elementary volume, the $\mathbf{L}$ matrix would be defined as:

$$
\left[\begin{array}{ll}
l_{11} & l_{12} \\
l_{21} & l_{22}
\end{array}\right]
$$

where $l_{11}$ and $l_{22}$ are the partial self inductances that are constant during the motion while $l_{12}$ and $l_{21}$ are the partial mutual inductances [30] that depend on the relative position and orientation of both the fixed and moving volumes. $\mathbf{R}(\mathbf{C}(t))$ is the resistance matrix and $\mathbf{K}(\mathbf{C}(t), \dot{\mathbf{C}}(t))$ takes into account the electromotive force due to the motional effects. In particular $\dot{\mathbf{C}}(t)$, termed as the derivatives of the system configuration at the instant $t$, describes the velocity of every elementary volume in the hypothesis of rigid body. The $\dot{\mathbf{C}}_{i}(t)$ corresponding to the $i$-th elementary volume is constituted by the three components of the translation velocity, the three components of the angular velocity, and the three coordinates of the center of rotation. It is worth to observe that $\dot{\mathbf{C}}_{i}(t) \equiv 0$ 
for elementary volumes belonging to the fixed body while for all the volumes belonging to the rigid body $\dot{\mathbf{C}}_{i}(t)$ is the same.

Given the two elementary volumes $j$ and $i$ we define their relative configuration $\mathbf{C}_{j \leftrightarrow i}(t)$ as the position and orientation of the $j$-th elementary volume with respect to the $i$-th. The position is obtained by expressing the coordinate of the center of gravity of the $j$-th volume in the coordinate system attached to the $i$-th one, while the orientation is given by the three Euler angles which define the angular position of the coordinate system attached to the $j$-th volume with respect coordinate system on the i-th one:

$$
\mathbf{C}_{j \leftrightarrow i}(t)=\left(x_{j, i}(t), y_{j, i}(t), z_{j, i}(t), \phi_{j, i}(t), \theta_{j, i}(t), \psi_{j, i}(t)\right) \quad i=1, \ldots, N_{f}+N_{m}
$$

Similarly we can define the derivative of the relative configuration $\dot{\mathbf{C}}_{j \leftrightarrow i}(t)$ by taking the derivatives of the first three elements of $\mathbf{C}_{j \leftrightarrow i}(t)$ and the derivatives of the three Euler angles respectively. It is indeed worth to observe that $\dot{\mathbf{C}}_{j \leftrightarrow i}(t) \equiv 0$ if $i$ and $j$ identify elementary volumes both belonging to the same (fixed or moving) body.

$\mathbf{F}$ and $\mathbf{T}$ are respectively the resultant force and torque acting on the moving rigid body, $m$ is the mass and $\mathbf{I}_{\theta \theta}$ is the inertia tensor. The three components vectors $\mathbf{q}=\left(x_{G}, y_{G}, z_{G}\right)$ and $\boldsymbol{\omega}=\left(\omega_{x}, \omega_{y}, \omega_{z}\right)$ respectively indicate the coordinates of the center of mass of the moving body and its angular velocity. It is worth to note that $\mathbf{F}$ and $\mathbf{T}$ are functions of $\mathbf{C}(t)$ and of the currents on the elementary volumes.

In the described formulation (as summarized in Appendix A), in correspondence to a given configuration, we firstly evaluate the dynamic interactions between couples of elementary volumes (one on the fixed and one on the moving body) in the hypothesis of unit current on them, so obtaining the force coefficients $\phi_{k j}$ which are function of the configuration. Subsequently we evaluate the effective force on the elementary volumes which constitute the moving body by considering the effective currents flowing on them $\mathbf{f}_{k j}=i_{k} \phi_{k j} i_{j}$ where $k$ spans the elementary volumes of the moving body and $j$ spans the ones belonging to the fixed body. By summing the effects of all this terms we can calculate the resultant force on the $k$-th elementary volume of the moving body:

$$
\mathbf{F}_{k}=\sum_{j=1}^{N_{f}} \mathbf{f}_{k j} \quad k=N_{f}+1, \ldots, N_{f}+N_{m}
$$

We can assert that the set of $\mathbf{F}_{k}$ represents the force distribution on the rigid body. The calculation of the resultant force on it is straightforward, as well as the resultant torque, once a pivot has been defined, e.g. the center of mass of the body.

\subsection{An elementary case: the jumping ring}

In order to make clearer the approach of the described formulation, and to better explain the underlying physics of the problems dealt with, a simple application example is here described with the aim of putting into evidence the physical meaning of (1).

Figure 1 shows the jumping ring. A conducting ring placed over the extended core of a vertical solenoid will jump in the air when the primary circuit is connected to an ac power line. In order to obtain the simplest governing equations we will assume a cylindrical symmetry for the device and an impressed sinusoidal current $j(t)$ on the fed coil. We also assume that the current in the jumping ring is uniformly distributed in its (rectangular) cross section and assume that the core is made of non-magnetizable material. This last assumption greatly reduces the device performance, but further simplifies the governing equations [26,27]. Under these hypotheses the electrical equilibrium equation (corresponding to (1a)) is written as:

$$
L \frac{d}{d t} i(t)+R i(t)+\frac{1}{S_{r}} \int_{V_{r}} \mathbf{v}(t) \times \mathbf{B}(t) \cdot \mathbf{u}_{\varphi} d v+M \frac{d}{d t} j(t)=0
$$

Where $L$ is self induction coefficient of the jumping ring, $R$ is its resistance, $M$ is the mutual induction coefficient between the jumping ring and the primary coil, $i(t)$ is the current induced in 


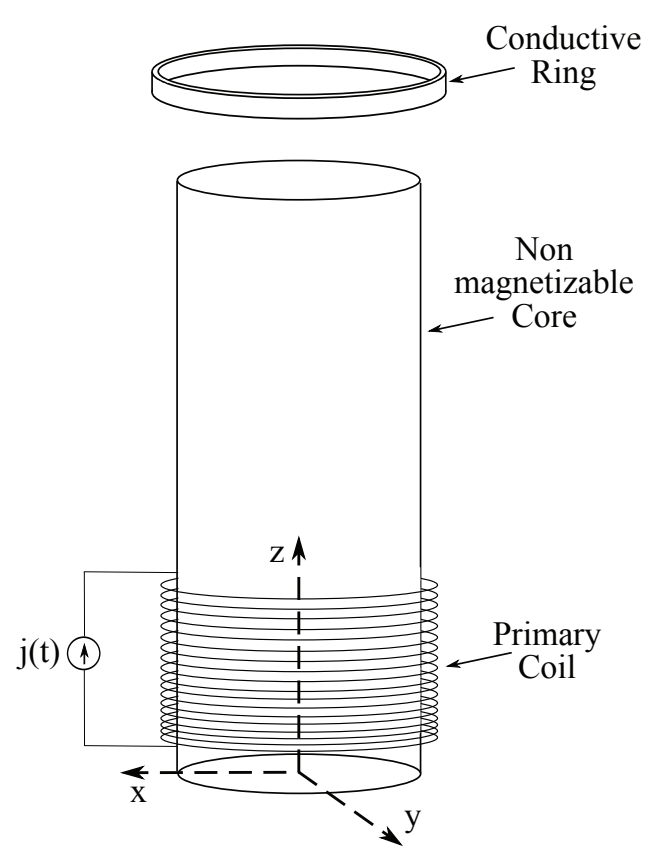

Figure 1. The jumping ring.

the conductive ring, $\mathbf{v}(t)$ is the velocity of the ring, $\mathbf{B}(t)$ is the magnetic flux density inside it and $\mathbf{u}_{\varphi}$ is the unit vector of the azimuth direction. The integral term in the equation can be considered as the motional electro-motive force (emf) evaluated along circumferences inside the ring and averaged on the cross section ( $V_{r}$ and $S_{r}$ denote the volume occupied by the ring and its cross section).

$\mathbf{B}(t)$ is due to the currents in both the primary and the jumping coils, but only the one on the primary contributes to the motional emf. Moreover taking into account the assumed symmetries

$$
\mathbf{v}(t)=\mathbf{u}_{z} v_{z}(t)=\mathbf{u}_{z} \dot{z}
$$

where $\mathbf{u}_{z}$ is the axial (z-axis) direction unit vector and $\dot{z}=v_{z}$ is the speed of the jumping ring that is the same on all the domain $V_{r}$, while the angular velocity is identically zero. As a result we can write:

$$
\frac{1}{S_{r}} \int_{V_{r}} \mathbf{v}(t) \times \mathbf{B}(t) \cdot \mathbf{u}_{\varphi} d v=v_{z} \frac{1}{S_{r}} \int_{V_{r}} B_{r}(t) d v=v_{z} H j(t)
$$

where $H=\frac{1}{S_{r}} \int_{V_{r}} B_{r}^{(1)}(t) d v$, with $B_{r}^{(1)}(t)$ being the magnetic flux density produced by the current in the primary coil when it has unit values, i.e. $j(t)=1$. Thus, the governing equation can be written as:

$$
L \frac{d}{d t} i(t)+R i(t)+v_{z} H j(t)+M \frac{d}{d t} j(t)=0
$$

In the above equation the coefficients $H$ and $M$ are function of the relative position between the two coils: $M=M(z(t))$ and $H=H(z(t))$ and the whole motional term can be written as

$$
v_{z} H(z(t)) j(t)=K(z(t), \dot{z}(t)) j(t)
$$

Arranging the currents in a two component vector we finally write:

$$
\left[\begin{array}{ll}
L & M(z(t))
\end{array}\right] \frac{d}{d t}\left[\begin{array}{l}
i(t) \\
j(t)
\end{array}\right]+\left[\begin{array}{ll}
R & K(z(t), \dot{z}(t))
\end{array}\right]\left[\begin{array}{l}
i(t) \\
j(t)
\end{array}\right]=0
$$


which is formally identical to (1a). Because of the assumed symmetries equation (1c) is useless, while equation (1b) reduces to:

$$
F_{z}(z(t))=m_{r} \ddot{z}(t)
$$

where $\ddot{z}$ is the acceleration of the jumping ring and $F_{z}(z(t))=\int_{V_{r}} J_{r} \mathbf{u}_{\varphi} \times \mathbf{B} \cdot \mathbf{u}_{z} d v$.

$J_{r}=i / S_{r}$ is the current density uniformly distributed in the ring, while $\mathbf{B}$ is the total magnetic flux density in the ring domain. Under the hypothesis of rigid body, since the integral is extended on the entire ring, we can consider the $\mathbf{B}$ produced by the primary coil only.

The force on the ring can by finally written as:

$$
F_{z}(z(t))=i(t) \phi(z(t)) j(t)
$$

This simple example allows understanding the difficulties of the numerical treatment. In fact the governing equations constitutes a set of nonlinear time-varying differential equations (which, for more complex problems, can reach the order of several thousands). The coefficients of the equations depend on the relative positions which are not assigned in advance.

Since integral formulations are time consuming during the coefficient matrix evaluation, it is worthy to investigate time integration schemes which require as few as possible coefficients evaluations. This simple example is well suited to make comparisons with other formulations, as the ones based on the finite element method. If FEM formulations are used to study systems with moving parts, they present the problem of the coupling between the meshes attached to the bodies in motions. Even if several methods (Lagrange multiplier [31,32], Minkowsky transform [33], used in commercial codes EFFE [20], MagNet [21], COMSOL [22]) are available, the more general ones require high computational times while the more efficient ones are not enough general.

In this particular case, when using a FEM formulation, the axial symmetry permits to build a 2D model of the device, where not only the active parts have to be meshed but also the air enclosing the device. In the case of study, the force exerted implies a relative motion with an increasing distance, meaning that the size of the block of air to be meshed, has to be wide.

The number of equations becomes significantly bigger than the ones used by the shown integral formulation also if in presence of skin effect. In this particular case, with the same hypothesis of axial symmetry, it is only necessary to divide the ring in a sufficient number of coaxial layers. The number of the equations (and of unknowns) will then be equal to the number of layers.

If the hypothesis of axial symmetric geometry is not valid anymore, the proposed method remains actually invariant: it is only needed to add mechanical equations that govern the rotations and translations in the other two directions, but the electrical equations remain the same. This is not true in the case of FEM formulations, where the problems becomes full 3D: the complexity increases in terms of number of equations and of computational time.

\subsection{The coupling}

During the dynamic evolution of the system, the relative configurations (and their derivatives) of the elementary volumes in relative motion can vary. As a consequence the coefficients matrices in (1) change with time. Integration of the system is complicated by its intrinsic nonlinearity: the coefficients of (1a) depend on the relative positions and velocities of elementary volumes that, in the more general case, are not assigned but are evaluated by (1b) and (1c), where both the torque and the force are functions of the (unknown) currents.

A number of techniques have been recently proposed for the solution of this kind of coupled problems. When a strong coupling between the equations is required [34,35], iterative procedures based on Gauss-Seidel or to Newton-Raphson schemes are usually adopted. They rely on the efficient evaluation (also approximated) of a number of derivatives [8,35,36]. These schemes when applied to the problem at hand may require very long computations [34].

Staggered solution strategies are very often used $[37,38]$, because they are characterized by low computation costs and produce acceptable results. In the case of magnetic levitation systems based on permanent magnets, which as known are characterized by unstable dynamics, it is needed to 
evaluate the unstable behavior. The evolution of this behavior has positive exponential dynamics, thus requiring more robust methods than the staggered ones. Starting from the simple weak (staggered) coupling of the equations, we propose two schemes characterized by an increasing degree of coupling. The scheme number 2 is equivalent to the strong approach.

The rationale behind the proposed schemes is the search for an approximation of the time dependence of the coefficients in (1a) and of the torque and force in (1b) and (1c) respectively. The predictor-corrector approach is used to obtain an approximate behavior of the named quantities by a linear interpolation between the known values at the previous time step and the predicted values at the next time step. Inserting this knowledge in the equations has the effect of considering updated values of the coefficients in (1a) and of torque and force in (1b) and (1c), so allowing a coupling between the equations which is stronger than the one in the staggered scheme. Integration is performed by implicit method (trapezoidal rule) in order to keep a larger time step.

\subsection{Weak coupling}

Let us assume as known the electrical and the mechanical quantities at the instant $t_{n}$, i.e. the currents and the position and velocity for every elementary volume, namely $\mathbf{i}\left(t_{n}\right)$ and $\mathbf{C}\left(t_{n}\right)$ and $\dot{\mathbf{C}}\left(t_{n}\right)$ (the knowledge of the configuration of the system and of its derivatives is equivalent to the knowledge of the position and velocity for every elementary volume).

We start integrating the electrical equations (1a) assuming that the coefficient matrices do not change in the interval $\left[t_{n}, t_{n+1}\right]$. Let $\mathbf{L}_{n}, \mathbf{R}_{n}$ and $\mathbf{K}_{n}$ be the matrices evaluated at $t_{n}$. The time interval $\left[t_{n}, t_{n+1}\right]$ has been subdivided in $m$ subintervals whose length $\delta t=\Delta t / \Delta t m m=$ $\left(t_{n+1}-t_{n}\right) /\left(t_{n+1}-t_{n}\right) m m$ is chosen according to the waveform of $e(t) . \delta t$ will be termed as the electric time constant. A Single Step Time Marching Algorithm (SSTMA) [39-41],characterized by time step $\delta t$ is used to integrate (1a) on the interval $\left[t_{n}, t_{n+1}\right]$, with $k=0, \ldots, m-1$ :

$$
\begin{aligned}
& \boldsymbol{\alpha}_{n, k}=\left(\mathbf{L}_{n}+\theta \cdot \delta t\left(\mathbf{R}_{n}+\mathbf{K}_{n}\right)\right)^{-1} \mathbf{e}\left(t_{n, k}\right)-\left(\mathbf{R}_{n}+\mathbf{K}_{n}\right) \mathbf{e}\left(t_{n, k}\right) \\
& \mathbf{i}\left(t_{n, k+1}\right)=\mathbf{i}\left(t_{n, k}\right)+\boldsymbol{\alpha}_{n, k} \delta t
\end{aligned}
$$

where $t_{n, k}=t_{n}+k \delta t$, and $\alpha_{n, k}$ can be interpreted as the average speed of variation if the currents in the $k$-th subinterval of the $n$-th interval. The $\theta$ value permits of realizing the known schemes represented in Table I (Galerkin or Crank-Nicolson are usually adopted).

$$
\begin{array}{|c|c|}
\text { ine } \theta & \text { Scheme } \\
\text { ine } 1 & \text { backward differences } \\
2 / 3 & \text { Galerkin } \\
1 / 2 & \text { Crank-Nicolson (trapezoidal rule) } \\
0 & \text { forward differences } \\
\text { ine } &
\end{array}
$$

Table I. Integration schemes varying $\theta$

In order to integrate (1b) and (1c), we consider $\boldsymbol{\Phi}\left(t_{n}\right)$ the matrix of the force coefficients at $t_{n}$ (as described in Appendix A), and evaluate the forces and the torques corresponding to the currents at the instant $t_{n}: \mathbf{F}\left(t_{n}\right)$ and $\mathbf{T}\left(t_{n}\right)$. Finally these values of force and torque, assumed to be constant on the time step, are used to integrate the rigid body equations (1b) and (1c).

Once the position is calculated, it is necessary to recalculate the matrices $\mathbf{L}, \mathbf{R}$ and $\mathbf{K}$ because the configuration has changed. This is typically a time consuming activity. In order to reduce the computational burden we defined a strategy with the aim of reducing the number of coefficient matrices evaluations. 
Given the relative configurations at two instants $\mathbf{C}_{j \leftrightarrow i}\left(t_{1}\right)$ and $\mathbf{C}_{j \leftrightarrow i}\left(t_{2}\right)$ we can define a two components vector $\boldsymbol{\Delta} \mathbf{C}_{j \leftrightarrow i, 1,2}=\left(r_{1,2} / r_{1}, \gamma_{1,2}\right)$ where

$$
\begin{aligned}
r_{1,2} & =\left(\left(x_{j, i}\left(t_{2}\right)-x_{j, i}\left(t_{1}\right)\right)^{2}+\left(y_{j, i}\left(t_{2}\right)-y_{j, i}\left(t_{1}\right)\right)^{2}+\left(z_{j, i}\left(t_{2}\right)-z_{j, i}\left(t_{1}\right)\right)^{2}\right)^{\frac{1}{2}} \\
r_{1} & =\left(x_{j, i}^{2}\left(t_{1}\right)+y_{j, i}^{2}\left(t_{1}\right)+z_{j, i}^{2}\left(t_{1}\right)\right)^{\frac{1}{2}} \\
\gamma_{1,2} & =\left(\left(\phi_{j, i}\left(t_{2}\right)-\phi_{j, i}\left(t_{1}\right)\right)^{2}+\left(\theta_{j, i}\left(t_{2}\right)-\theta_{j, i}\left(t_{1}\right)\right)^{2}+\left(\psi_{j, i}\left(t_{2}\right)-\psi_{j, i}\left(t_{1}\right)\right)^{2}\right)^{\frac{1}{2}}
\end{aligned}
$$

$\Delta \mathbf{C}_{j \leftrightarrow i, 1,2}$ can be considered as an indicator of the variation of the relative position and of the orientation of the $j$-th elementary volume w.r.t. the $i$-th one in the time interval $\left[t_{1}, t_{2}\right]$, i.e. how much the volumes have relatively moved (one with respect to the other).

The matrix $\Delta \mathbf{C}_{1,2}$ is built with all the $\Delta C_{j \leftrightarrow i, 1,2}$ :

$$
\Delta \mathbf{C}_{1,2} \triangleq\left[\Delta \mathbf{C}_{j \leftrightarrow i, 1,2}\right]
$$

It is worth to notice that it has sense to build this matrix only for $i$ spanning elementary volumes in the fixed body and $j$ spanning elements in the moving body.

The updating of $\mathbf{L}, \mathbf{R}$ and $\mathbf{K}$ is performed by adopting an auxiliary matrix $\Delta \mathbf{D}$ (of the same size as $\boldsymbol{\Delta} \mathbf{C}_{1,2}$ ) which is managed as follows. At time $t_{0}$ all its elements are initialized as:

$$
\Delta \mathbf{D}_{j \leftrightarrow i, 0}=\Delta \mathbf{C}_{j \leftrightarrow i, 0,0}=(0,0)
$$

while at the subsequent time steps:

$$
\Delta \mathbf{D}_{j \leftrightarrow i, n+1}=\Delta \mathbf{D}_{j \leftrightarrow i, n}+\Delta \mathbf{C}_{j \leftrightarrow i, n, n+1}
$$

At the instant $t_{n+1}$, we look for the elements of $\Delta \mathrm{D}$ which have at least one of the two components greater than a fixed threshold; then we set to $(0,0)$ these elements and update the corresponding entries of the $\mathbf{L}, \mathbf{R}$ and $\mathbf{K}$ matrices.

In the authors' experience supported by several numerical experiments, a threshold of $\Delta \mathbf{D}_{\text {threshold }}=(0.03,0.05 \mathrm{rad})$ does not significantly affect the accuracy of the simulations while providing an important computational time saving. Increasing the threshold to $(0.06,0.1 \mathrm{rad})$ provides acceptable results; some results varying the threshold values will be shown on Section 3.

\subsection{Scheme 1: weak coupling with correction}

When the velocity increases this simple scheme needs to be improved: this is accomplished by the introduction of a prediction-correction algorithm in the integration of the mechanical equations. The integration of the electrical equation remains unchanged. In this new scheme, the mechanical equations are prior integrated by using $\mathbf{F}\left(t_{n}\right)$ and $\mathbf{T}\left(t_{n}\right)$ as driving terms, obtaining at $t_{n+1}$ a prediction of the configuration and of its derivative, denoted by $\tilde{\mathbf{C}}\left(t_{n+1}\right)$ and $\dot{\tilde{\mathbf{C}}}\left(t_{n+1}\right)$ respectively. In the following, the superscript ${ }^{\sim}$ will be used to denote predicted quantities. Force and torque are evaluated again by using the force coefficients in the predicted configuration $\tilde{\boldsymbol{\Phi}}\left(t_{n+1}\right)$ and in correspondence of the currents $\mathbf{i}\left(t_{n+1}\right): \tilde{\mathbf{F}}\left(t_{n+1}\right)$ and $\tilde{\mathbf{T}}\left(t_{n+1}\right)$. Finally the mechanical equations are integrated again by using the trapezoidal rule, assuming that the force and torque acting on the moving body assume a linear behavior in the interval $\left[t_{n}, t_{n+1}\right]$ between the values $\mathbf{F}\left(t_{n}\right), \mathbf{T}\left(t_{n}\right)$ and the values $\tilde{\mathbf{F}}\left(t_{n+1}\right), \tilde{\mathbf{T}}\left(t_{n+1}\right)$. The corrected value of the position of the moving body at $t_{n+1}$ is then obtained. This prediction-correction scheme requires two evaluations of the forces and torques; the computational overhead with respect to the scheme 1 is compensated by the increased precision. The algorithm above described for the updating of the elements of the matrices $\mathbf{L}, \mathbf{R}$ and $\mathbf{K}$ is adopted in this case too. Figure 2 shows a detailed flowchart of the considered integration scheme. 


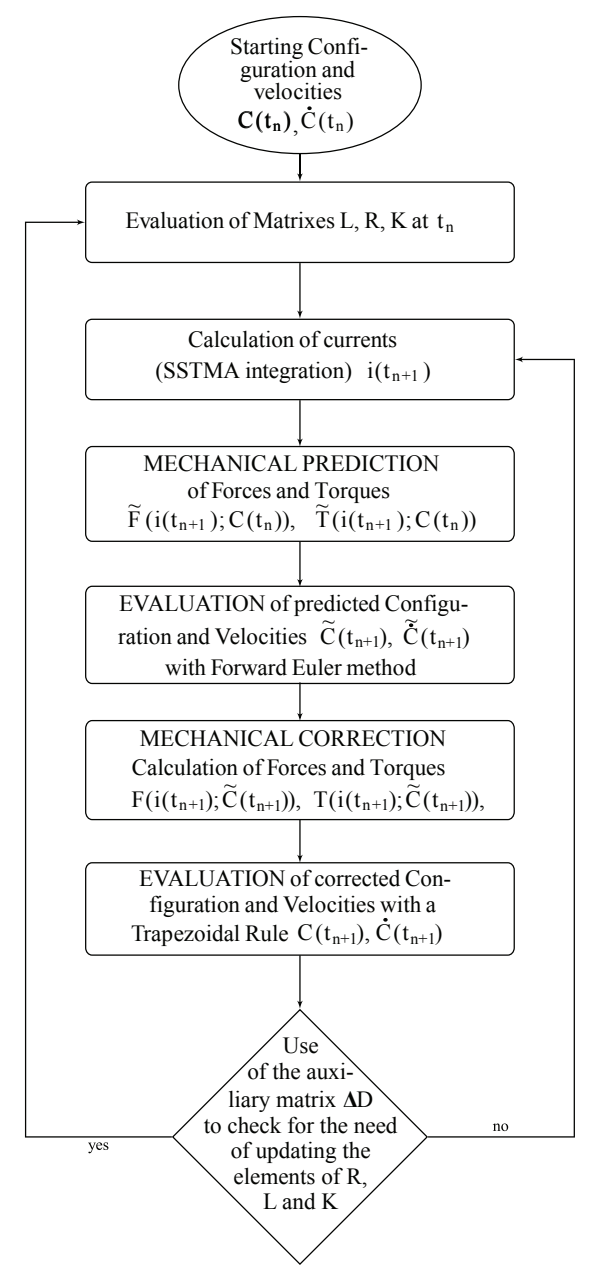

Figure 2. Flowchart of the integration scheme 1.

\subsection{Scheme 2: stronger coupling by interleaved predictor-corrector.}

In the weak coupling approach, the electrical equations are integrated in the interval $\left[t_{n}, t_{n+1}\right]$ considering the mechanical quantities (position and velocity) as constants and related to the configuration $\mathbf{C}\left(t_{n}\right)$ and its derivative $\dot{\mathbf{C}}\left(t_{n}\right)$ at the instant $t_{n}$. Similarly in the integration of the mechanical equation we use the force and the torque obtained by the force coefficients matrix $\boldsymbol{\Phi}\left(t_{n}\right)$ and the currents $\mathbf{i}\left(t_{n}\right)$. Scheme 1 uses updated values of the torque and of the force in the integration of the mechanical equations by using a predictor-corrector scheme while the electromagnetic equations are treated as before.

In the analysis of devices characterized by high speed (i.e. in which the rate of change of the electrical parameters due to the motion is comparable with the rate of change of the electrical quantities) the hypothesis of considering constant the elements of $\mathbf{L}, \mathbf{R}$ and $\mathbf{K}$ during the time step is not acceptable, unless using very short time steps, with increased computational times. A stronger coupling between equations in (1) would require updating the matrices during the integration of (1a). A new integration scheme is presented with the aim of increasing the coupling between equations in (1) while maintaining acceptable length of the time integration step. The main idea besides this scheme is to properly approximate the evolution of the coefficients matrices $\mathbf{L}, \mathbf{R}$ and $\mathbf{K}$ during the integration step in order to take into account their variation during the motion. More specifically a piecewise linear behavior obtained by a predictor-corrector scheme is assumed. 


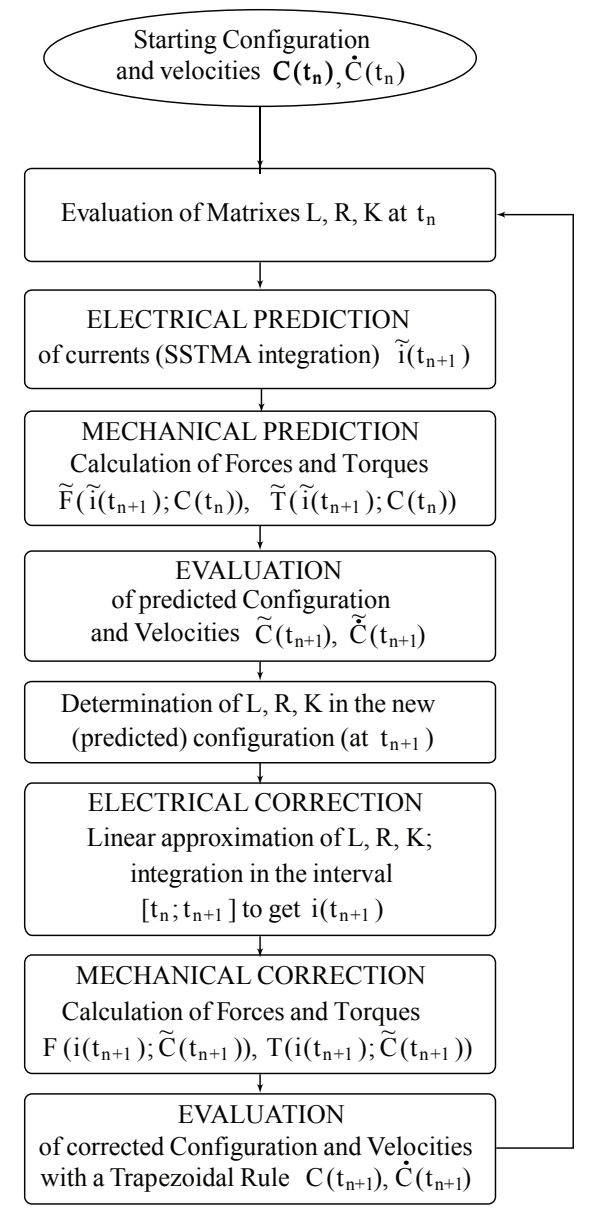

Figure 3. Flowchart of the integration scheme 2.

Let $\Delta t$ be the time step. We start the proposed procedure by performing the operations as in the scheme 1 until the prediction of the configuration $\tilde{\mathbf{C}}\left(t_{n+1}\right)$ and of its derivative $\dot{\tilde{\mathbf{C}}}\left(t_{n+1}\right)$; namely: a) integration of the electrical equations (1a) by SSTMA and b) integration of the mechanical equations (1b) and (1c) by the Forward Euler formula.

Then we estimate the derivatives of $\mathbf{L}, \mathbf{R}$ and $\mathbf{K}$ in $\left[t_{n}, t_{n+1}\right]$ as:

$$
\begin{aligned}
\dot{\mathbf{L}}\left(t_{n}\right) & \simeq \frac{\mathbf{L}\left(\tilde{\mathbf{C}}\left(t_{n+1}\right)\right)-\mathbf{L}\left(\tilde{\mathbf{C}}\left(t_{n}\right)\right)}{\Delta t} \\
\dot{\mathbf{R}}\left(t_{n}\right) & \simeq \frac{\mathbf{R}\left(\tilde{\mathbf{C}}\left(t_{n+1}\right)\right)-\mathbf{R}\left(\tilde{\mathbf{C}}\left(t_{n}\right)\right)}{\Delta t} \\
\dot{\mathbf{K}}\left(t_{n}\right) & \simeq \frac{\mathbf{K}\left(\tilde{\mathbf{C}}\left(t_{n+1}\right), \dot{\tilde{\mathbf{C}}}\left(t_{n+1}\right)\right)-\mathbf{K}\left(\tilde{\mathbf{C}}\left(t_{n}\right), \dot{\tilde{\mathbf{C}}}\left(t_{n}\right)\right)}{\Delta t}
\end{aligned}
$$

and write the piecewise linear approximations in the interval $\left[t_{n}, t_{n+1}\right]$ :

$$
\begin{aligned}
& \mathbf{L}(t) \simeq \mathbf{L}\left(t_{n}\right)+\dot{\mathbf{L}}\left(t_{n}\right) \cdot\left(t-t_{n}\right)=\mathbf{L}_{n}+\dot{\mathbf{L}}_{n} \cdot\left(t-t_{n}\right) \\
& \mathbf{R}(t) \simeq \mathbf{R}\left(t_{n}\right)+\dot{\mathbf{R}}\left(t_{n}\right) \cdot\left(t-t_{n}\right)=\mathbf{R}_{n}+\dot{\mathbf{R}}_{n} \cdot\left(t-t_{n}\right) \\
& \mathbf{K}(t) \simeq \mathbf{K}\left(t_{n}\right)+\dot{\mathbf{K}}\left(t_{n}\right) \cdot\left(t-t_{n}\right)=\mathbf{K}_{n}+\dot{\mathbf{K}}_{n} \cdot\left(t-t_{n}\right)
\end{aligned}
$$

These expressions are introduced in (1a): 


$$
\left(\mathbf{L}_{n}+\dot{\mathbf{L}}_{n} \cdot\left(t-t_{n}\right)\right) \frac{d \mathbf{i}}{d t}+\left[\left(\mathbf{R}_{n}+\dot{\mathbf{R}}_{n} \cdot\left(t-t_{n}\right)\right)+\left(\mathbf{K}_{n}+\dot{\mathbf{K}}_{n} \cdot\left(t-t_{n}\right)\right)\right] \mathbf{i}=\mathbf{e}(t) \quad t \in\left[t_{n}, t_{n+1}\right]
$$

The time interval $\left[t_{n}, t_{n+1}\right]$ is subdivided in $m$ subintervals whose length $\delta t=\Delta t / m=\left(t_{n+1}-\right.$ $\left.t_{n}\right) / m$ is chosen according to the waveform of $e(t)$ (e.g. if $e(t)$ is a sinusoid with period $T$, than $\delta t=T / 20$ can be an acceptable choice). $\delta_{t}$ will be termed as the electric time constant. Integrating (17) on the $k$-th subinterval yields:

$$
\begin{aligned}
& \int_{t_{n}+k \delta t}^{t_{n}+(k+1) \delta t}\left(\mathbf{L}_{n}+\dot{\mathbf{L}}_{n}\left(t-t_{n}\right)\right) \frac{d \mathbf{i}}{d t} d t+\ldots \\
& \ldots+\int_{t_{n}+k \delta t}^{t_{n}+(k+1) \delta t}\left[\left(\mathbf{R}_{n}+\dot{\mathbf{R}}_{n}\left(t-t_{n}\right)\right)+\left(\mathbf{K}_{n}+\dot{\mathbf{K}}_{n}\left(t-t_{n}\right)\right)\right] \mathbf{i} d t=\int_{t_{n}+k \delta t}^{t_{n}+(k+1) \delta t} \mathbf{e}(t) d t
\end{aligned}
$$

A numerical integration (trapezoidal-like) is used:

$$
\begin{aligned}
& \mathbf{L}_{n}\left(\mathbf{i}\left(t_{n}+(k+1) \delta t\right)-\mathbf{i}\left(t_{n}+k \delta t\right)\right)+\frac{\delta t \dot{\mathbf{L}}_{n}}{2}\left(\mathbf{i}\left(t_{n}+(k+1) \delta t\right)+\mathbf{i}\left(t_{n}+k \delta t\right)\right)+ \\
& \quad+\frac{\delta t\left(\mathbf{R}_{n}+\mathbf{K}_{n}\right)}{2}\left(\mathbf{i}\left(t_{n}+(k+1) \delta t\right)+\mathbf{i}\left(t_{n}+k \delta t\right)\right)+\frac{\delta^{2} t\left(\dot{\mathbf{R}}_{n}+\dot{\mathbf{K}}_{n}\right)}{2} \mathbf{i}\left(t_{n}+(k+1) \delta t\right)= \\
& \quad=\frac{\delta t}{2}\left(\mathbf{e}\left(t_{n}+(k+1) \delta t\right)+\mathbf{e}\left(t_{n}+k \delta t\right)\right)
\end{aligned}
$$

Collecting terms (and defining $\left.\mathbf{i}_{n, k+1}=\mathbf{i}\left(t_{n}+(k+1) \delta t\right)\right)$ we can write:

$$
\begin{aligned}
\left(\mathbf{L}_{n}\right. & \left.+\frac{\delta t \dot{\mathbf{L}}_{n}}{2}+\frac{\delta t\left(\mathbf{R}_{n}+\mathbf{K}_{n}\right)}{2}+\frac{\delta^{2} t\left(\dot{\mathbf{R}}_{n}+\dot{\mathbf{K}}_{n}\right)}{2}\right) i_{n, k+1}= \\
& =\frac{\delta t}{2}\left(\mathbf{e}_{n, k+1}+\mathbf{e}_{n, k}\right)+\left(\mathbf{L}_{n}-\frac{\delta t \dot{\mathbf{L}}_{n}}{2}-\frac{\delta t\left(\mathbf{R}_{n}+\mathbf{K}_{n}\right)}{2}\right) i_{n, k}
\end{aligned}
$$

Starting form $k=0$ and iterating until $k=m-1$ we obtain the corrected values of $\mathbf{i}\left(t_{n+1}\right)$. Force and torque are evaluated again by using the force coefficients in the predicted configuration $\tilde{\Phi}\left(t_{n+1}\right)$ and the currents just obtained.

The integration of the mechanical equations, as in the scheme 2, yields the corrected position of the moving body. The flowchart of the proposed scheme is reported in Fig. 3.

The coefficients of $\Delta t^{2}$ could be discarded, so only the evaluation of the derivatives of $\mathbf{L}$ is needed to set up the proposed scheme. However the evaluation of the derivatives of $\mathbf{R}$ is trivial, while the evaluation of the derivatives of $\mathbf{K}$ can be obtained with a little overhead when evaluating the predicted force coefficient matrix.

\subsection{Extension to deformable bodies}

In the adopted formulation, as described in [26-28], the evaluation of the resultant force and torque, acting on the moving body, is performed by integrating $\mathbf{J} \times \mathbf{B}$ and $(\mathbf{J} \times \mathbf{B}) \times \overrightarrow{\mathbf{P}-\mathbf{O}}$ terms respectively. In the torque expression $\mathbf{P}$ represents the application point of the force on the elementary volume (that coincides with its center of mass) and $\mathbf{O}$ is the pivot. In case of magnetizable body (with assumed uniform magnetization) the current density is equivalent to the $\mathbf{M} \times \mathbf{n}$ term and the integration domain is the surface of the magnetized elementary volume.

In the term $\mathbf{J} \times \mathbf{B}$ the magnetic flux density can be considered as the sum of two terms: one due to the currents in the moving object $\mathbf{B}_{\text {mov }}$ and one due to the current in the body at rest $\mathbf{B}_{\text {rest }}$. As 


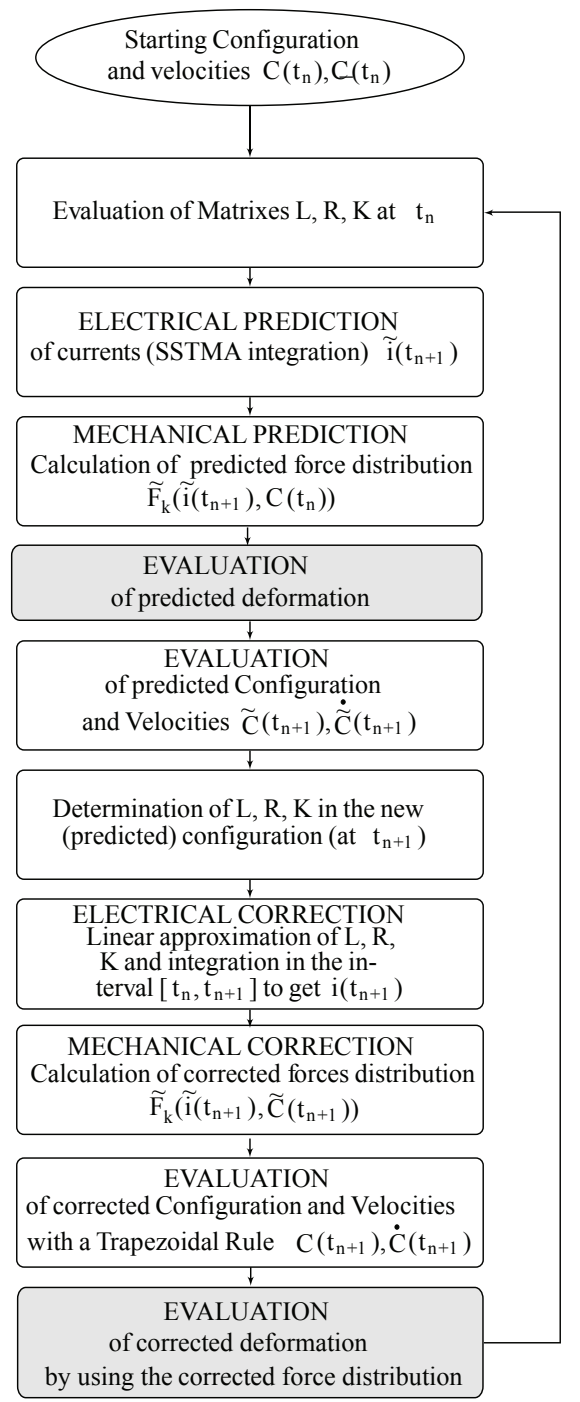

Figure 4. Flowchart of the integration scheme 2.1.

known, in order to address the solution of the problem of the motion of the rigid body, only the term $\mathbf{B}_{\text {rest }}$ has to be evaluated since the resultant of the (internal) forces due to $\mathbf{B}_{\text {mov }}$ is zero. It is worth to note that, using the adopted formulation, it is possible to determine the total force distribution acting on conductive and magnetizable bodies, as it may be required to solve elasticity problem. This force distribution data can be properly exported to a software able to solve the elasticity problems such as Ansys [42] or Adina [43].

If the hypothesis of linear elasticity can be assumed, the deformed configuration can be obtained by a simple post processing activity by using the force distribution in the non deformed configuration. If deformations are too large, not only the hypothesis of linear elasticity cannot be assumed, but also their impact on the coefficients matrices of the electrical equation has to be taken into account. If fracture and sliding of portions of volumes with respect to others are absent, we can insert two calls to the elasticity solver in the integration scheme 2, obtaining the scheme 2.1 as shown in Fig. 4, where the two gray-shaded boxes indicate these calls.

The first one performs the evaluation of the predicted deformations by using the values of the predicted forces distribution. The predicted configuration is then obtained by adding the displacements evaluated under the hypothesis of rigid body with the displacements due to the deformations. The second call to the elasticity solver is performed in order to evaluate the corrected 
deformation of by using the corrected forces distribution. The corrected configuration is finally obtained composing the rigid body movement with the corrected deformation.

The deformed configuration has to be imported in the electro-mechanical software preserving the shape of the elementary volumes. This implies some degree of approximation in the deformed geometry. In order to obtain the elementary volumes in the deformed configuration the following procedure can be used.

For every elementary volume:

- Evaluate the position of its vertices after the deformation; the new volume may not belong to the set of admissible elementary volumes.

- Build the a new admissible elementary volume by fitting the position of its vertices on the one of the deformed volume, preserving its size.

Under the hypotheses of absence of fractures and absence of sliding contacts, these procedure preserves the equivalent network connectivity, and all the considerations reported in this paper and in [26] apply as well.

\section{VALIDATION}

The validation of computer codes is usually performed by comparison with experimental data, analytical solutions or numerical results produced by other codes. The first two modes are preferred but, for these kind of systems, their availability is limited or even absent. The transient electromechanical analysis is a nonlinear problem (forces and torques are functions of the position); at the best of the authors' knowledge analytical solutions are not available. A number of benchmark problems known as TEAM (Testing Electromagnetic Analysis Method) problems have been proposed in the past years in the more general context of computational electromagnetics. The TEAM problems involving moving conductors are the numbers 9, 17 and 28. In problem 9 a moving body with given constant velocity is considered [44], while for problem 17 no measured data are available [45]. The problem number 28 is a transient problem with electromechanical coupling and measured data are available [46].

The geometry of problem 28 is reported in Fig. 5(a), showing a circular plate located above two hollow cylindrical coils. The inner coil has $N_{1}=960$ and the outer one has $N_{2}=576$ turns. The dimensions of the device are reported in Fig. 5(b).

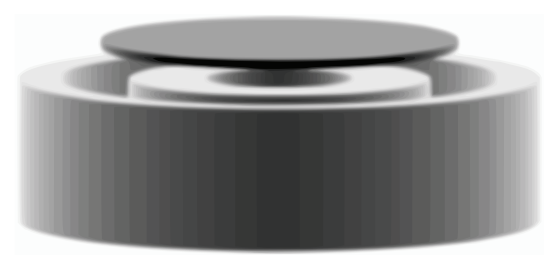

(a) 3D view of the TEAM problem 28, courtesy of [46].

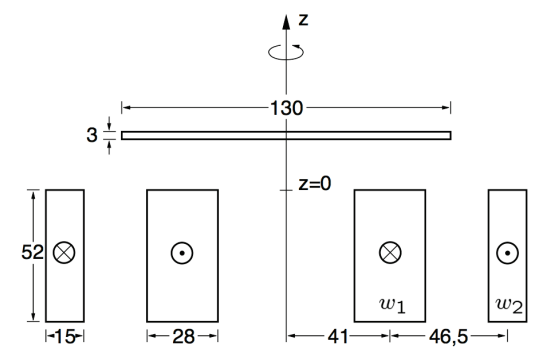

(b) Device dimensions (mm), courtesy of [46].

Figure 5. Team 28 Problem.

Both coils are fed with the same sinusoidal current but with reversed direction:

$$
i(t)=I_{M} \sin \left(2 \pi f_{0} t\right) \quad t \geq 0, \quad I_{M}=20 \mathrm{~A}, \quad f_{0}=50 \mathrm{~Hz}
$$

Transients in the current waveform are suppressed by a proper synchronization with the driving supply voltage and the possible reaction with the eddy currents on the plate are neglected; the 


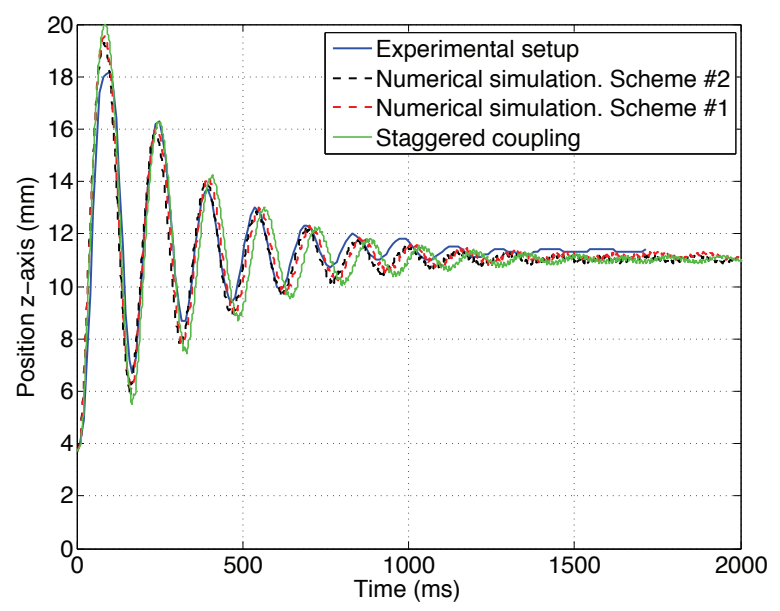

Figure 6. Comparison between measured and computed results.

currents in the coils can be considered as impressed. Due to the induced eddy currents a repulsive force is exerted on the plate, which after some dumped oscillations attains a stationary position characterized by a gap of $11.3 \mathrm{~mm}$ between the plate and the upper edge of the coils. The initial gap is $3.8 \mathrm{~mm}$.

A number of simulations have been run on this geometry by using the discussed integration schemes. The schemes 1 and 2 outperform the weak coupling scheme since this usually requires very small time steps to obtain acceptable accuracy. For this reason it will be no more considered in the following. Let $\Delta t_{1}$ and $\Delta t_{2}$ be the time steps chosen for the scheme 1, and 2 respectively. Considering the overhead of the scheme 2 with respect to the scheme 1, in order to compare them keeping approximatively the same execution times, we choose $\Delta t_{2}=2 \Delta t_{1}$.

We initially considered the system characterized by axisymmetric geometry with one degree of freedom. Fig. 6 reports the comparison between the measured data (the gap between the plate and the coils) and the results of the simulations for the two schemes under comparison. In the same figure is also shown the waveform obtained by using the staggered method with the same time step as scheme 1 . Scheme 1 and scheme 2 exhibit a very good accuracy showing at the steady condition a difference of less than $2 \%$ with respect to the measured data. The near coincidence of the numerical results by the two integration schemes is due to the low speed (of the order of tenths of meters per second) involved. The used threshold is $(0.03,0.05 \mathrm{rad})$. The time step $\Delta t_{2}$ was $8 \mathrm{~ms}$ while, due to the frequency of the feeding currents the electrical time step $\delta t$ was $1 \mathrm{~ms}$. The scheme 2 took about 90 min to run on a GPU based desktop. The scheme 1 took about 140 min, while the staggered method took 120 minutes, but the results were less accurate.

Figure 7 show the differences obtained in the results, when modifying the $\Delta \mathbf{D}_{\text {threshold }}$ values. As already told, a threshold of $\boldsymbol{\Delta} \mathbf{D}_{\text {threshold }}=(0.03,0.05 \mathrm{rad})$ does not significantly affect the accuracy of the simulations. Increasing the threshold to $(0.06,0.1 \mathrm{rad})$ provides still acceptable results. The error continues to increase increasing the threshold to $(0.1,0.15 \mathrm{rad})$. As for the simulation time it was roughly inversely proportional to the adopted threshold.

We also validated the code considering a 6 DoF problem obtained by altering the cylindrical symmetry of problem 28 . The plate has been rotated of -3 degrees with respect to a barycentric axis parallel to the y direction and moved by its initial position by a displacement of $5 \mathrm{~mm}$ (along the $\mathrm{x}$ axis direction) and of $-3 \mathrm{~mm}$ (along the $\mathrm{y}$ axis). Because of the absence of experimental data, we performed a self consistency analysis of the obtained results. We started observing that the stationary position of the plate (at the end of the mechanical transients) is coincident with that obtained by considering the one degree of freedom behaviour: the initial position of the six degrees of freedom simulation is within the region of asymptotic stability of the stationary configuration. In order to check for the correctness of the solution, we considered the time evolution of the resultant forces $\left(F_{x, G}(t), F_{y, G}(t), F_{z, G}(t)\right)$ and torques $\left(T_{x, G}(t), T_{y, G}(t), T_{z, G}(t)\right)$ on the plate, and 


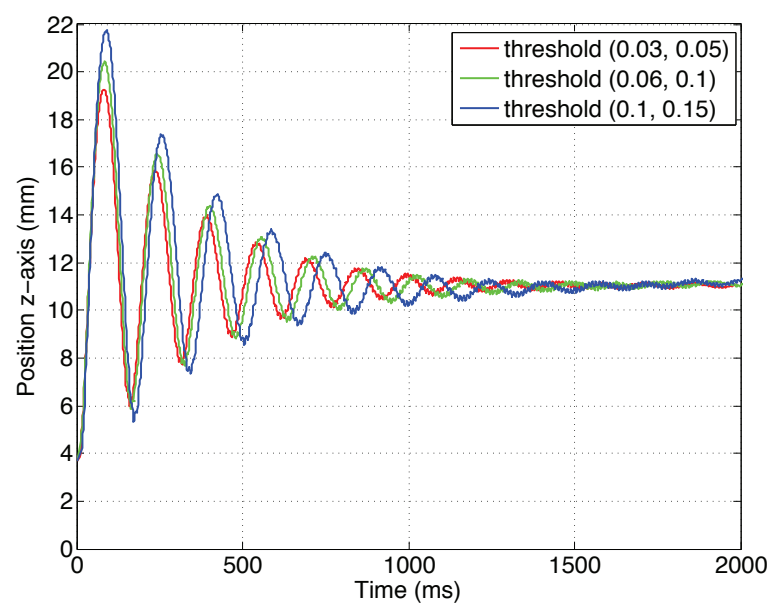

Figure 7. Comparison with varying the $\Delta \mathbf{D}_{\text {threshold }}$ values.

of the description of the motion of the plate in terms of the positions of the center of gravity $\left(x_{G}(t), y_{G}(t), z_{G}(t)\right)$ and of the rotations of the plate respect to a barycentric frame attached to the moving body $\left(\phi_{G}(t), \theta_{G}(t), \psi_{G}(t)\right)$.

By using MSC ADAMS (a commercial code for rigid body dynamic analysis) [47] we integrated the motion equations of the plate when subjected to $\left(F_{x, G}(t), F_{y, G}(t), F_{z, G}(t)\right)$ and $\left(T_{x, G}(t), T_{y, G}(t), T_{z, G}(t)\right)$; we obtained a close replica of $\left(x_{G}(t), y_{G}(t), z_{G}(t)\right)$ $\left(\phi_{G}(t), \theta_{G}(t), \psi_{G}(t)\right)$.

The comparison is reported in Figs. 8 and 9 respectively. Figure 8 shows the comparison between the two lateral components of the velocity of the center of gravity of the plate, while Fig. 9 shows the comparison between angular velocities in the same axes.

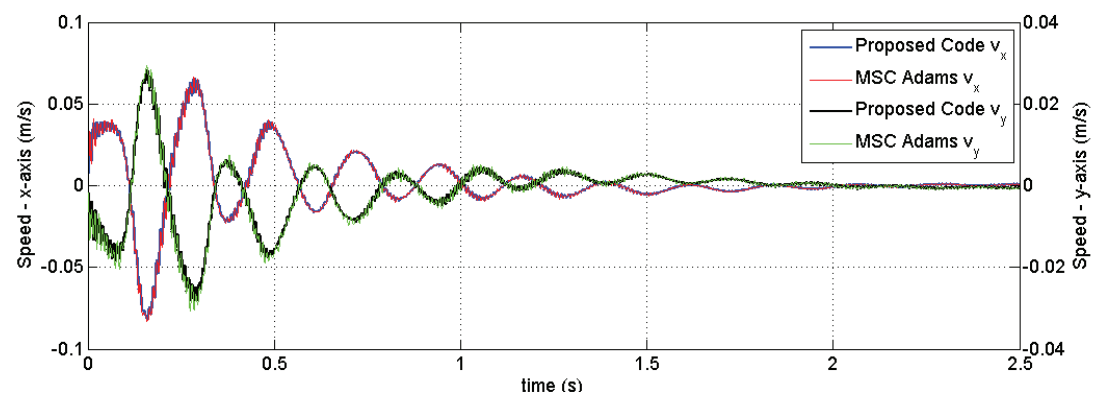

Fig. 8. Comparison of the velocities of the center of gravity.

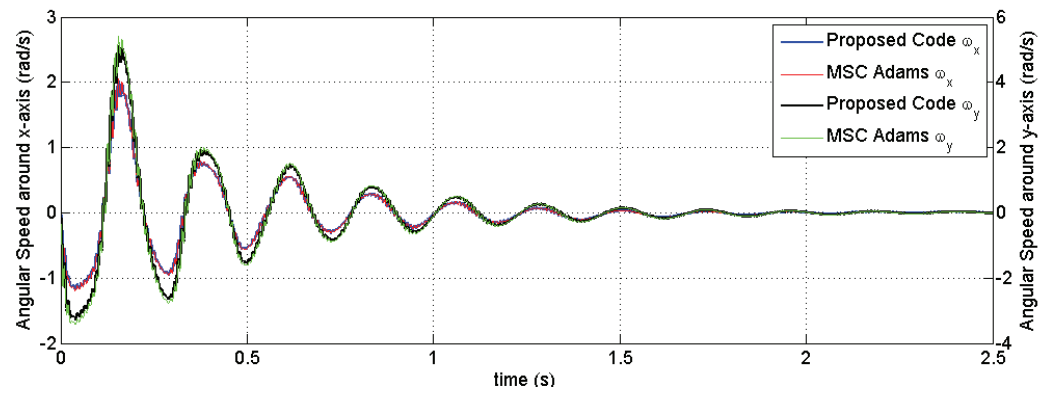

Fig. 9. Comparison of the angular velocities.

Conversely, we forced the plate to move according to the trajectory described by $\left(x_{G}(t), y_{G}(t), z_{G}(t)\right)$ and $\left(\phi_{G}(t), \theta_{G}(t), \psi_{G}(t)\right)$ obtained by our code. We simulated the evolution 


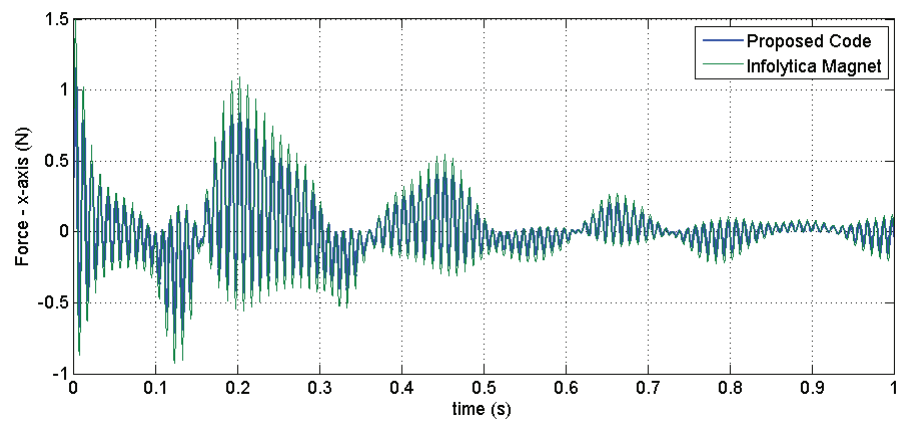

Fig. 10. Comparison of the force along the x-axis.

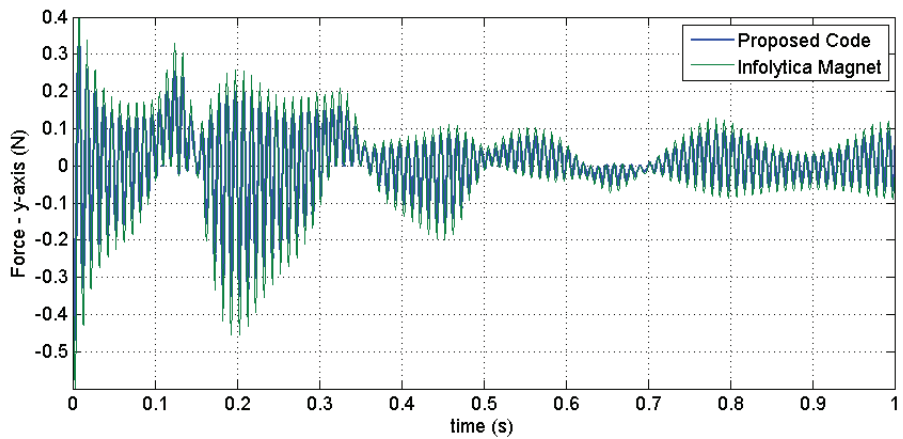

Fig. 11. Comparison of the force along the y-axis.

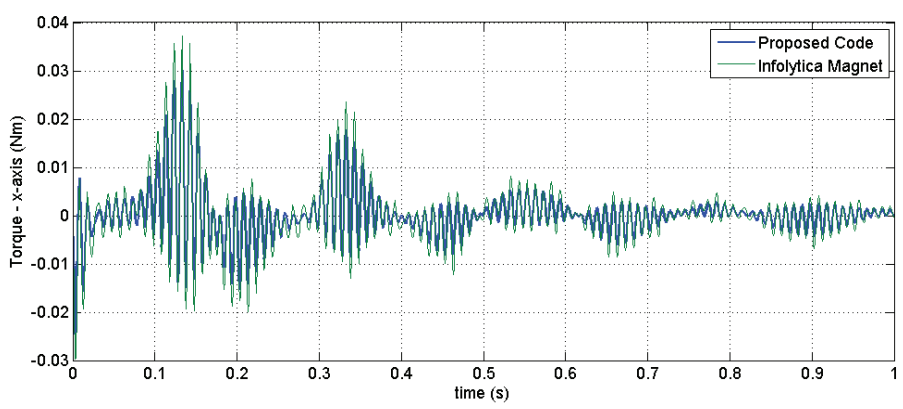

Fig. 12. Comparison of the torque along the $\mathrm{x}$-axis.

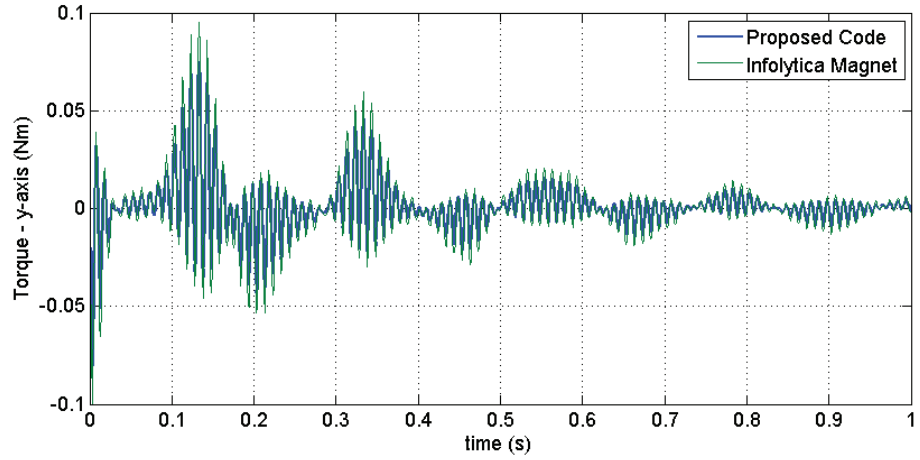

Fig. 13. Comparison of the torque along the y-axis.

of the device by using the commercial code Infolytica MAGNET [21] evaluating the resultant force and torque on the plate. The obtained results were in excellent agreement as shown in Figs.10-13. 
The results here shown are produced by scheme 1. Those by scheme 2 are almost indistinguishable. As expected, all the waveforms are characterized by the presence of an oscillation at $100 \mathrm{~Hz}$. Only 1 second of the evolution is shown in order to preserve the visibility of the images.

\section{APPLICATION EXAMPLE}

As an example of application of the code, we considered a test model which, in our opinion, could be useful for the validation of numerical formulations for electromechanical problems with more than one degree of freedom.

The analyzed device is an electrodynamical bearing [48-52] consisting of a magnetic rotor that levitates over a conducting sheet with an initial angular speed. The motion of the magnets induces eddy currents in the track that simultaneously create lift and drag forces.

The device is shown on Fig. 14. The rotor is an Halbach array with 4 pole-pairs. The parameters of the model are shown on Table II.

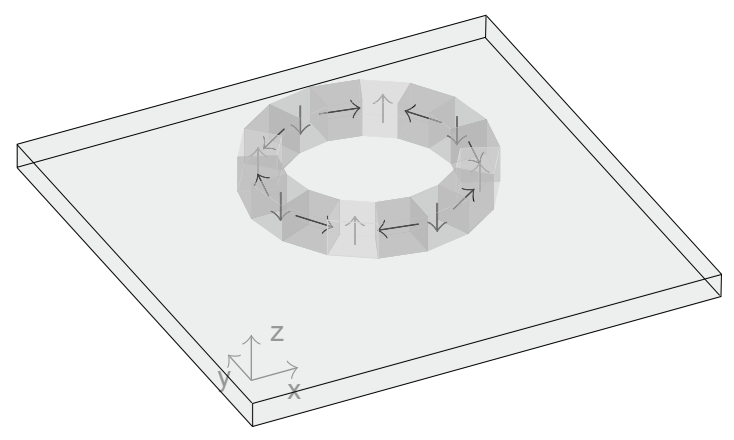

Figure 14. Electrodynamical bearing.

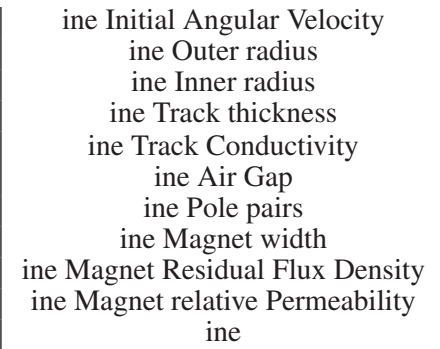

\begin{tabular}{|c|}
$628 \mathrm{rad} / \mathrm{s}$ \\
$0.05 \mathrm{~m}$ \\
$0.035 \mathrm{~m}$ \\
$0.004 \mathrm{~m}$ \\
$59.6 \times 10^{6} 1 /(\mathrm{m} \Omega)$ \\
$0.004 \mathrm{~m}$ \\
4 \\
$0.015 \mathrm{~m}$ \\
$1.36 \mathrm{~T}$ \\
1.2
\end{tabular}

Table II. Parameters of the model

The rotor is driven at the angular speed of $628 \mathrm{rad} / \mathrm{s}$ and positioned above the conducting plate. The distance between the center of gravity of the device and the upper side of the plate is $11.5 \mathrm{~mm}$. The device continues to rotate for a little more than 4 seconds. During this time the angular velocity is reduced by a drag torque, and so the lift force decreases, becoming smaller than gravity force, until the device hits the track. The initial time step was $\Delta_{t}=250 \mu \mathrm{s}$, while $\delta_{t}$ was $50 \mu \mathrm{s}$ (about 80000 electrical steps). As the angular speed was lowering, $\Delta t$ was increased until the value of $500 \mu \mathrm{s}$ at the end of the simulation. $\delta_{t}$ remained unchanged. The model took about 38 hours to run on a GPU based environment. Commercial codes available at our Department were not able to simulate this device. 


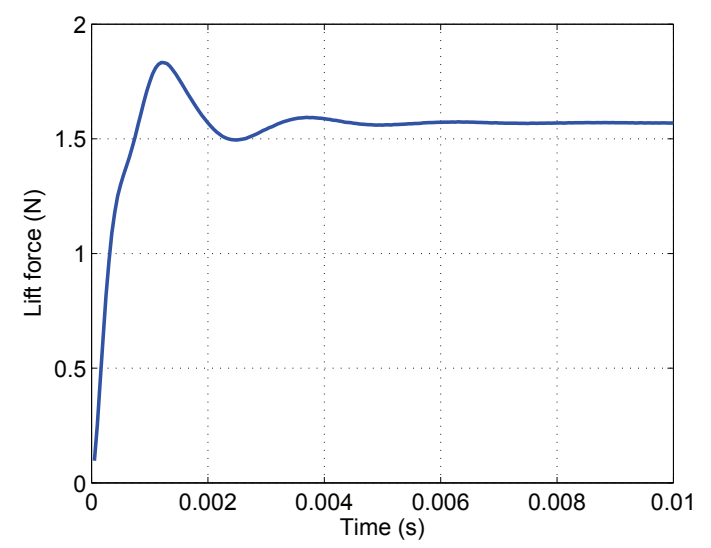

Figure 15. Initial force transient with device blocked on the z-axis.

Due to the initial imposed angular velocity of $628 \mathrm{rad} / \mathrm{s}$ (corresponding to a peripheral velocity of more than $30 \mathrm{~m} / \mathrm{s}$ ), the integration scheme 1 required a small time step w.r.t. the one of scheme 2 in order to achieve the same results. More precisely $\Delta t_{1} \simeq \Delta t_{2} / 3$.

For the first 0.01 seconds of the simulation, the device has been kept blocked along the z-axis and with constant angular speed, in order to allow the current transient to complete. In this phase, the spin device could only rotate around the z-axis. The initial force transient is shown in Fig. 15.

After this transient, the device has been kept free to move in the $6 \mathrm{DoF}$. The full evolution of the lift force and the drag torque is shown in Fig. 16 and Fig. 17 respectively. Fig. 18 reports the position on the z-axis of the center of mass of the spin device respect to a reference system fixed on the lower bottom of the track, while Fig. 19 shows the angular velocity around the $\mathrm{z}$-axis.

The values of the forces and the position of the device in the last steps of the simulation could be wrong because of the contact with compenetration of the rotor with the plane.

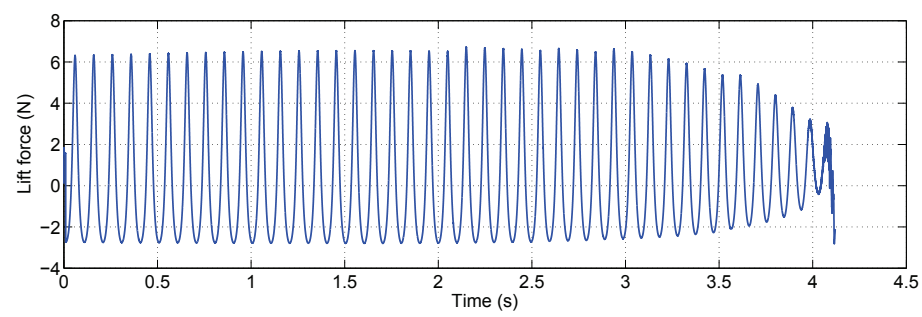

Figure 16. Lift Force.

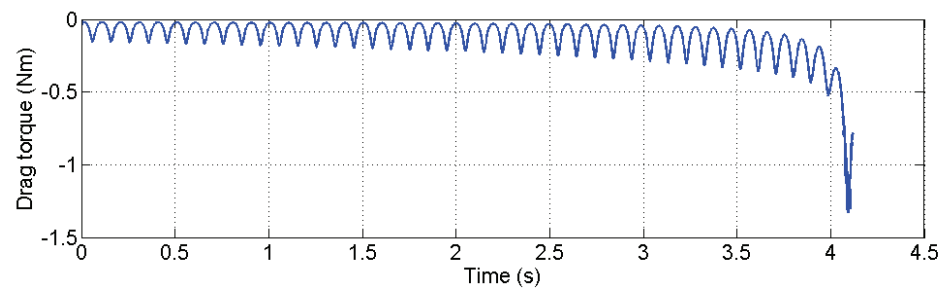

Figure 17. Drag Torque. 


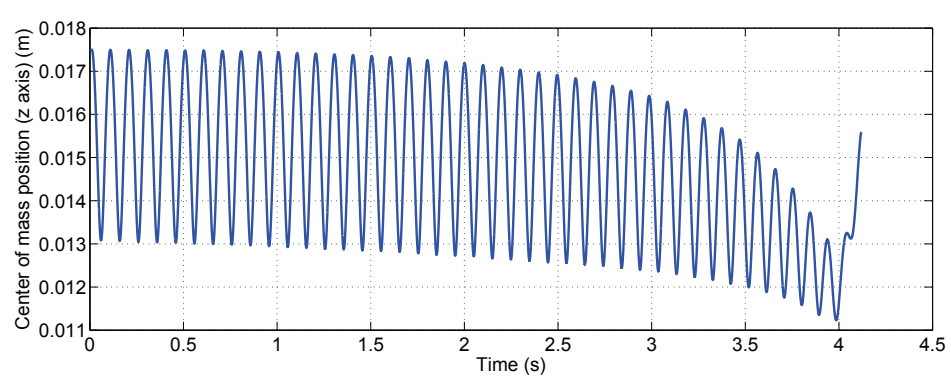

Figure 18. Position of the center of mass along the z-axis.

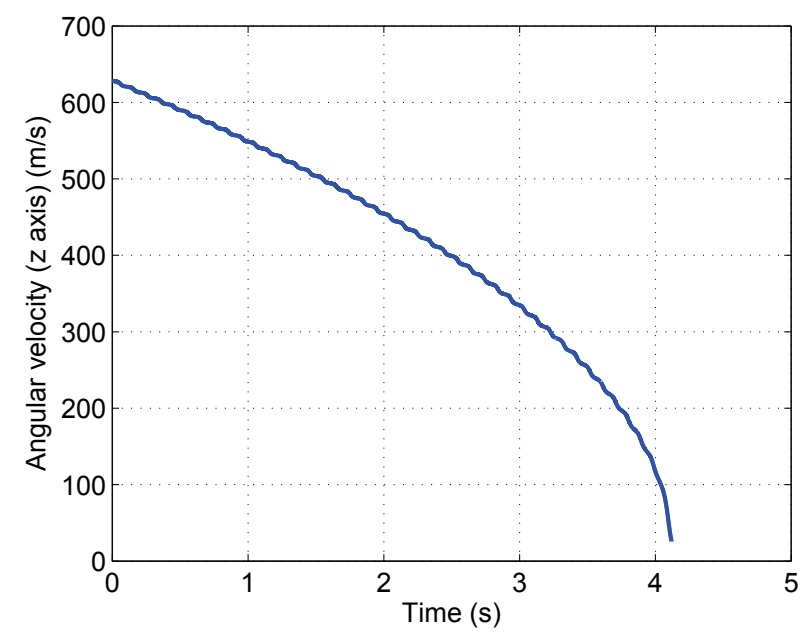

Figure 19. Angular velocity.

\section{CONCLUSION}

A new approach for the numerical solution of coupled electromechanical problems has been presented. Two valid integration schemes have been developed for problems in which simplifying hypotheses (weak electro mechanic coupling, absence of motional induced voltages, knowledge of the trajectory of the motion - i.e. one degree of freedom - ) cannot be assumed. For these problems the use of model order reduction techniques [53] could results unviable. The first scheme is characterized by a predictor-corrector approach applied to the mechanical equations. It shows good results if the simulated device speed is of some tenths of $\mathrm{m} / \mathrm{s}$. The second scheme consist of nested predictor corrector algorithms. It solves the mechanical and the electrical problems contextually. This scheme shows a good accuracy even with high speed devices. Both the methods have been validated by comparison with experimental data (when available) and with results obtained by other numerical formulations. Finally an example of application of a 2 degrees of freedom device has been modeled and simulated.

\section{A. GENERAL FORMULATION OF THE METHODOLOGY}

The approach for the solution of coupled electromagnetic and mechanical problems is based on the the low frequency formulation of the Maxwell equations and the hypothesis of rigid body, for what concerns the mechanical equations. For the sake of clarity, let us briefly recall the the differential form of the quasi static approximation of the Maxwell equations in Eq. (21) : 


$$
\begin{aligned}
\nabla \cdot \mathbf{D} & =\rho, & \mathbf{D} & =\varepsilon_{0} \varepsilon_{r} \mathbf{E}, \\
\nabla \cdot \mathbf{B} & =0, & \mathbf{B} & =\mu_{0} \mu_{r} \mathbf{H}, \\
\nabla \times \mathbf{E} & =-\frac{\partial \mathbf{B}}{\partial t}, & & \mathbf{J}=\sigma \mathbf{E} \\
\nabla \times \mathbf{H} & =\mathbf{J} & &
\end{aligned}
$$

where the universal constants appearing in the equations are the permeability of free space $\mu_{0}$ and the permittivity of free space $\varepsilon_{0} . \mu_{r}$ is the relative permeability, $\varepsilon_{r}$ the relative permittivity and $\sigma$ the conductivity. The sources are the electric charge density (charge per unit volume) $\rho$ and the electric current density (current per unit area) $\mathbf{J}$.

Regarding the equations of motion of the rigid body, in the Newton-Euler formulation, are expressed in terms of the angular velocity and acceleration vectors. It is also assumed that the origin of the body coordinate system (reference point) is the center of mass of the body. Using this assumption and the relationships between the angular velocity and acceleration vectors, and the orientation coordinates, it can be shown that [54] the dynamics equation can be written as:

$$
\begin{aligned}
m \ddot{\mathbf{q}} & =\mathbf{F} \\
\mathbf{I}_{\theta \theta} \dot{\boldsymbol{\omega}} & =\mathbf{T}-\boldsymbol{\omega} \times \mathbf{I}_{\theta \theta} \boldsymbol{\omega}
\end{aligned}
$$

where $\ddot{\mathrm{q}}$ is the linear acceleration vector, $\dot{\omega}$ and $\boldsymbol{\omega}$ are respectively the angular acceleration and velocity vectors defined in the global coordinate system, $\mathrm{m}$ is the mass of the rigid body, $\mathbf{I}_{\theta \theta}=\mathbf{A} \overline{\mathbf{I}}_{\theta \theta} \mathbf{A}^{T}$ is the inertia tensor defined in the global coordinate system - with $\mathbf{A}$ defined as the rotation matrix describing the body orientation dynamics from the initial condition using Euler angles notation, and $\overline{\mathbf{I}}_{\theta \theta}$ the (constant) inertia tensor defined in the body coordinate system -, and $\mathbf{T}$ is the vector of Cartesian moments that act on the rigid body.

Let us now consider a system of two bodies that magnetically interact. For the sake of simplicity, we refer to a system composed of a fixed and a moving body. A local coordinate system is defined for both the bodies. The two bodies, both constituted of conductive and linear magnetizable materials, are discretized in elementary volumes. Only slab volumes are considered here; the use of cylindrical sectors is discussed in [23] and [55]. Connecting the centers of nearby elements we obtain a 3D structured grid, as shown in Fig. 20.

Let now observe the segments $l_{p_{x}}, l_{p_{y}}$ ed $l_{p_{z}}$ that join the points of the reticulum in the cartesian axis directions. For every of these segments we can define a new elementary volume with two faces that cross the ends of the segment and with the segment height, and with edges oriented as the three cartesian direction, as shown in Fig. 21, evidenced in pink.

Let $N$ be the number of conductive elements obtained. Current density $J$ is nonzero only in the direction of the segment and is assumed to be uniformly distributed. Uniform distribution of the magnetization $\mathbf{M}$ is assumed in the $M$ elementary volumes obtained by the discretization of the magnetizable materials. Ohms law in the generic point $P$ of the $k$-th elementary volume can be written as:

$$
\rho \mathbf{J}_{k}(P, t)=\mathbf{E}_{k}(P, t)+\mathbf{v}_{k}(P, t) \times \mathbf{B}_{k}(P, t)
$$

where $\rho$ is the resistivity, $\mathbf{J}_{k}(P, t)$ is the current density, $\mathbf{E}_{k}(P, t)$ is the electric field, $\mathbf{B}_{k}(P, t)$ is the magnetic flux density and $\mathbf{v}_{k}(P, t)$ is the relative velocity between the conductor and the magnetic flux density distribution. Since $\nabla \cdot \mathbf{B}_{k}(P, t)=0$, we can write $\mathbf{B}_{k}(P, t)=\nabla \times$ $\mathbf{A}_{k}(P, t)$, being the vector potential; considering that $\nabla \times\left(\mathbf{E}_{k}(P, t)+\frac{\partial}{\partial t} \mathbf{A}_{k}(P, t)\right)=0$ we can write $E_{k}(P, t)+\frac{\partial}{\partial t} A_{k}(P, t)=-\nabla V_{k}(P, t)$, and substituting in the Ohms law, yelds:

$$
\rho \mathbf{J}_{k}(P, t)=-\nabla V_{k}(P, t)-\frac{\partial \mathbf{A}_{k}(P, t)}{\partial t}+\mathbf{v}_{k}(P, t) \times \mathbf{B}_{k}(P, t)
$$

Constitutive equation at the generic point $P$ inside the $h$-th magnetizable elementary volume is written in terms of the magnetization: 


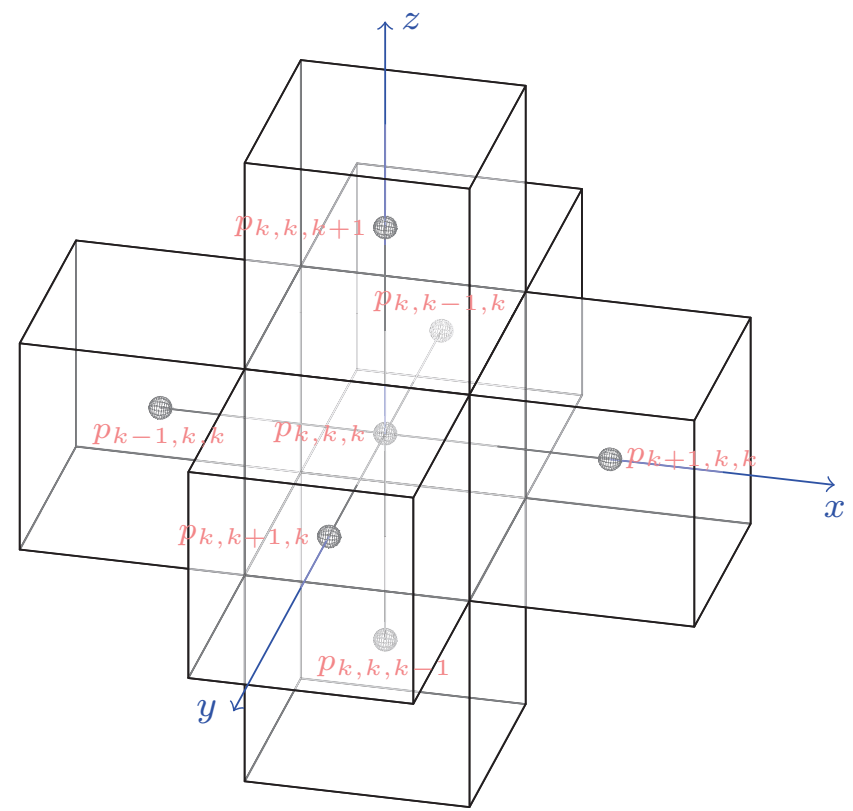

Figure 20. The 3D structured grid.

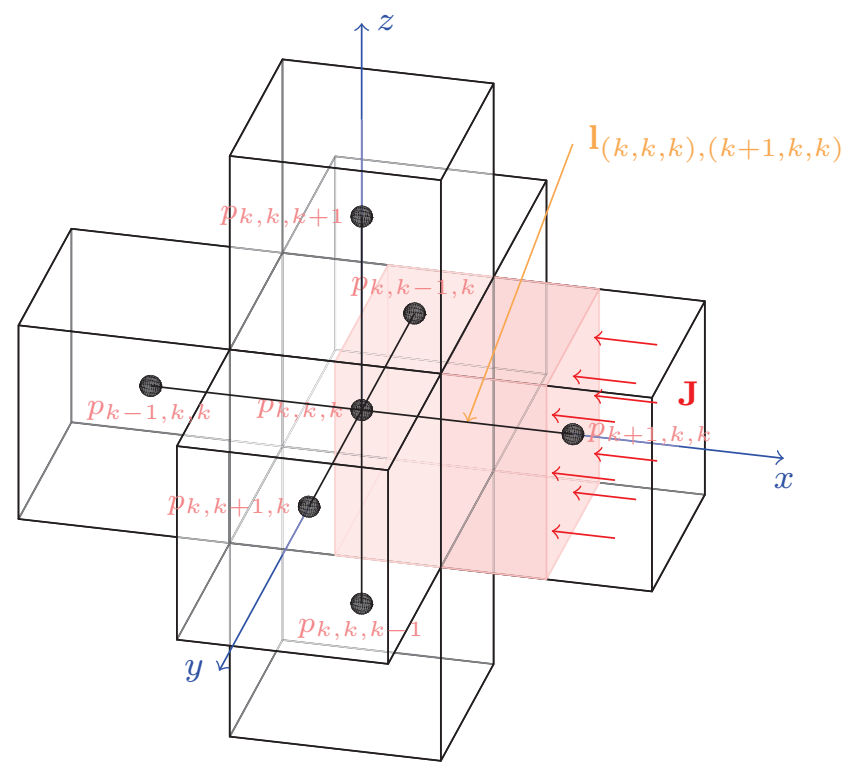

Figure 21. Definition of the new elementary volume.

$$
\mathbf{H}_{h}\left(\mathbf{B}_{h}(P)\right)=\frac{\mathbf{B}_{h}(P)}{\mu_{0} \mu_{r}}=\frac{\mathbf{B}_{h}(P)}{\mu_{0}}-\mathbf{M}_{h}(P)
$$

where $\mathbf{H}_{h}=\mathbf{B}_{h}(P) / \mu_{0} \mu_{r}$ is the linear characteristic of the material in the $h$-th slab, with $\mu_{0}$ the magnetic permeability of vacuum and $\mu_{r}$ the relative magnetic permeability of the material respectively. Time does not appear in (25) since it is an algebraic equation. The magnetic flux density in (24) and (25) can be written as follows: 


$$
\mathbf{B}_{h}(P, t)=\sum_{j=1}^{N} \boldsymbol{\beta}_{(P), h, j} \mathbf{J}_{j}(t)+\sum_{j=1}^{M} \boldsymbol{\alpha}_{(P), h, j} \mathbf{M}_{j}(t)
$$

where $\boldsymbol{\beta}_{(P), h, j}$ and $\boldsymbol{\alpha}_{(P), h, j}$ are $3 \times 3$ matrices functions of the source domain spanned by the index $j$ and of the position of the point $P$ inside the $h$-th volume. Because of the assumed uniform distribution of $\mathbf{M}$ and $\mathbf{J}$ on the chosen elementary volumes (slabs) the coefficients in (26) can be expressed by using analytical expressions. The vector potential $\mathbf{A}_{k}(P, t)$ can be expressed with a similar expression:

$$
\mathbf{A}_{k}(P, t)=\sum_{j=1}^{N} \lambda_{(P), k, j} \mathbf{J}_{j}(t)+\sum_{j=1}^{M} \vartheta_{(P), k, j} \mathbf{M}_{j}(t)
$$

Equation (24) is projected on the segments of the grid and is averaged on the cross section:

$$
\begin{aligned}
& R_{k}^{\prime} i_{k}(t)+\sum_{j=1}^{N} L_{k, j}^{\prime} \frac{d}{d t} i_{j}(t)+\sum_{j=1}^{N} K_{k, j}^{\prime} i_{j}(t)+ \\
& \quad+\sum_{j=1}^{3 M} \Gamma_{k, j} \frac{d}{d t} m_{j}(t)+\sum_{j=1}^{3 M} \Pi_{k, j} m_{j}(t)=u_{k}(t) \quad k=1, \ldots, N
\end{aligned}
$$

In $(28) i_{j}(t)$ is the current flowing in the $j$-th conductive elementary volume, $m_{j}(t)$ represent the equivalent surface magnetization current corresponding to one of the components of the magnetization vector (the total number of equivalent surface magnetization currents is $3 \mathrm{M}$ ). Finally $u_{k}(t)$ is the potential drop between the terminals of the segment due to the irrotational component of the electric field. This is the governing equation of a branch which is a series connection of a resistor, an inductor coupled with the inductors in other branches and controlled voltage generators that take into account the motional effects, as shown in Fig. 22.

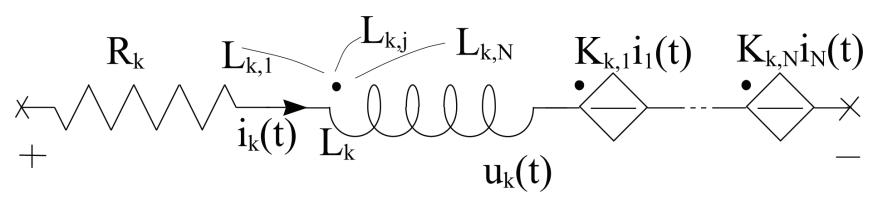

Figure 22. Two-terminals element configuration.

The last two sums in (28) take into account the effect of magnetized materials. The self and mutual inductance coefficients $L_{k, j}$ are obtained by integrating, on the $k$-th slab, the vector potential produced by a unit current flowing on the $j$-th slab. Obviously, the mutual induction coefficients between elements of the same body will be constant during the motion, while the ones between elements of different bodies will vary in time with relative position. This is still true for the motional terms that depend on the relative position of the volumes and will have to be update during the motion of the system. Analytical expressions are available to evaluate fields and potentials of the defined coefficients together with some closed formulas for the evaluation of the mutual coefficients.

Equation (25) is averaged on every elementary volume of the magnetic materials, yielding:

$$
\Omega \mathbf{m}=\Psi \mathbf{i}
$$

where $\boldsymbol{\Omega}$ and $\boldsymbol{\Psi}$ are respectively built with the coefficients $\boldsymbol{\beta}_{(P), h, j}$ and $\boldsymbol{\alpha}_{(P), h, j}$ defined in (26).

Equation (29) allows evaluating the magnetization currents as a function of the currents in the branches of the equivalent network; magnetization currents can be considered as imposed by a set of current generators driven by $i_{1}, \ldots, i_{N}$. In this context (29) can be considered as the control equation of these generators. The equivalent electric network defined by (28) and (29) is solved by the mesh analysis. The governing equations can be written as: 


$$
\mathbf{R}^{\prime} \mathbf{i}+\mathbf{L}^{\prime} \frac{d}{d t} \mathbf{i}+\mathbf{K}^{\prime} \mathbf{i}+\mathbf{\Gamma} \frac{d}{d t} \mathbf{m}+\mathbf{\Pi} \mathbf{m}=\mathbf{u}
$$

where $\mathbf{i}$ is the vector of the $n$ currents on the chords and $\mathbf{m}$ is the vector of the $3 M$ magnetization currents. Finally, making the $\mathbf{m}$ term explicit in equation (29):

$$
\mathbf{R i}+\mathbf{L} \frac{d}{d t} \mathbf{i}+\mathbf{K i}=\mathbf{u}
$$

Once currents are known the magnetic force acting on the $k$-th elementary volume can be evaluated by integrating $\mathbf{J}_{k}(P, t) \times \mathbf{B}_{k}(P, t)$ for conductive regions and $\left(\mathbf{M}_{k}(P, t) \times \mathbf{n}_{k}\right)_{S_{k}} \times$ $\mathbf{B}_{k}(P, t)$ for the magnetizable material, where $\left(\mathbf{M}_{k}(P, t) \times \mathbf{n}_{k}\right)_{S_{k}}$ represents the equivalent magnetization currents on the surface of the volume. The resultant force and torque are then inserted in the rigid body mechanical equations that allow updating the velocity and the position.

The values of the currents can be used to determine the force between $k$ and $j$ elements:

$$
\mathbf{f}_{k j}=\int_{\Gamma_{k}} \mathbf{J}_{k} \times \mathbf{B}_{j} d \Gamma=\phi_{k j} i_{k} i_{j} .
$$

where $\phi_{k j}$ is the force coefficient between $k$ and $j$, i.e. the force corresponding to unit currents on them. We hypothesize that these forces are applied on the center of mass of the elementary volumes.

\section{ACKNOWLEDGEMENT}

The authors would like to thank the NVIDIA's Academic Research Team for their equipment donation of two NVIDIA Tesla K20c GPUs that have been extensively exploited for the simulation activity.

\section{REFERENCES}

1. Ren Z, Razek A. A strong coupled model for analysing dynamic behaviours of non-linear electromechanical systems. Magnetics, IEEE Transactions on 1994; 30(5):3252-3255.

2. Hasirci U, Balikci A, Zabar Z, Birenbaum L. Concerning the design of a novel electromagnetic launcher for earthto-orbit micro-and nanosatellite systems. Plasma Science, IEEE Transactions on 2011; 39(1):498-503.

3. Hasirci U, Balikci A, Zabar Z. A novel magnetic-levitation system: Design, implementation, and nonlinear control. Plasma Science, IEEE Transactions on 2011; 39(1):492-497.

4. Engel TG, Veracka MJ. Solid-projectile helical electromagnetic launcher with variable gradient stator and magnetically levitated armature. Plasma Science, IEEE Transactions on 2011; 39(12):3371-3377.

5. Wu S, Yu C, Cui S, Song L, Liu M. Mechanical analysis and evaluation of compulsator. Plasma Science, IEEE Transactions on $2011 ; 39(1): 322-327$.

6. Andreykiv A, Rixen DJ. Numerical modelling of electromechanical coupling using fictitious domain and level set methods. International journal for numerical methods in engineering 2009; 80(4):478-506.

7. Joosten M, Dettmer W, Perić D. Analysis of the block gauss-seidel solution procedure for a strongly coupled model problem with reference to fluid-structure interaction. International Journal for Numerical Methods in Engineering 2009; 78(7):757-778.

8. Rochus V, Rixen DJ, Golinval JC. Monolithic modelling of electro-mechanical coupling in micro-structures. International journal for numerical methods in engineering 2006; 65(4):461-493.

9. Confalonieri F, Corigliano A, Dossi M, Gornati M. A domain decomposition technique applied to the solution of the coupled electro-mechanical problem. International Journal for Numerical Methods in Engineering 2013; 93(2): $137-159$.

10. Musolino A, Rizzo R, Tucci M, Matrosov VM. A new passive maglev system based on eddy current stabilization. Magnetics, IEEE Transactions on 2009; 45(3):984-987.

11. Earnshaw S. \{On the nature of the molecular forces which regulate the constitution of the luminiferous ether\}. Trans. Camb. Phil. Soc 1842; 7:97-112.

12. Braunbek W. Freischwebende körper im elektrischen und magnetischen feld. Zeitschrift für Physik 1939; 112(1112):753-763.

13. Schaaf Jr J, Zowarka Jr R, Davey K, Weldon J. The amt maglev test sled-eml weapons technology transition to transportation. Magnetics, IEEE Transactions on 1997; 33(1):379-383.

14. Putman PT, Salama K. Optimization of energy conversion in monolithic superconducting magnets. Applied Superconductivity, IEEE Transactions on $2003 ; 13(2): 2146-2149$.

15. Pichot M, Kajs J, Murphy B, Ouroua A, Rech B, Hayes R, Beno J, Buckner G, Palazzolo A. Active magnetic bearings for energy storage systems for combat vehicles. Magnetics, IEEE Transactions on 2001; 37(1):318-323.

16. Murphy B, Manifold S, Kitzmiller J. Compulsator rotordynamics and suspension design. Magnetics, IEEE Transactions on 1997; 33(1):474-479. 
17. Shearwood C, Williams C, Mellor P, Chang K, Woodhead J. Electro-magnetically levitated micro-discs. Microengineering Applications in Optoelectronics, IEE Colloquium on, IET, 1996; 6-1.

18. Hoburg JF. Modeling maglev passenger compartment static magnetic fields from linear halbach permanent-magnet arrays. Magnetics, IEEE Transactions on 2004; 40(1):59-64.

19. Peterson W. Numerical solution of eddy current problems in ferromagnetic bodies travelling in a transverse magnetic field. International journal for numerical methods in engineering 2003; 58(12):1749-1764.

20. Effe, bathwick electrical design limited 2012. URL http://www. bedl.co.uk/.

21. Infolytica magnet 2012. URL http://www. infolytica.com/en/products/magnet/.

22. Multiphysics C. Users guide. Version 2007; 4:290-298.

23. Musolino A, Rizzo R. Numerical modeling of helical launchers. Plasma Science, IEEE Transactions on 2011; 39(3):935-940.

24. Albanese R, Rubinacci G. Integral formulation for $3 \mathrm{~d}$ eddy-current computation using edge elements. IEE Proceedings A (Physical Science, Measurement and Instrumentation, Management and Education, Reviews) 1988; 135(7):457-462.

25. Codecasa L, Specogna R, Trevisan F. A geometric integral formulation for eddy-currents. International journal for numerical methods in engineering 2010; 82(13):1720-1736.

26. Musolino A, Rizzo R, Tripodi E, Toni M. Modeling of electromechanical devices by gpu-accelerated integral formulation. International Journal of Numerical Modelling: Electronic Networks, Devices and Fields 2012; .

27. Esposito N, Musolino A, Raugi M. Modelling of three-dimensional nonlinear eddy current problems with conductors in motion by an integral formulation. Magnetics, IEEE Transactions on 1996; 32(3):764-767.

28. Barmada S, Musolino A, Raugi M, Rizzo R. Analysis of the performance of a combined coil-rail launcher. Magnetics, IEEE Transactions on 2003; 39(1):103-107.

29. Musolino A, Rizzo R, Toni M, Tripodi E. Acceleration of numerical formulations by using graphic processing units and its application in electromagnetic launcher modeling. Plasma Science, IEEE Transactions on 2013; 41(5):11041111.

30. Paul CR. Inductance: loop and partial. John Wiley \& Sons, 2011.

31. Golovanov C, Coulomb JL, Marechal Y, Meunier G. 3d mesh connection techniques applied to movement simulation. Magnetics, IEEE Transactions on 1998; 34(5):3359-3362.

32. Marechal Y, Meunier G, Coulomb J, Magnin H. A general purpose tool for restoring inter-element continuity. Magnetics, IEEE Transactions on 1992; 28(2):1728-1731.

33. Rodger D, Lai H. A comparison of formulations for $3 \mathrm{~d}$ finite element modeling of electromagnetic launchers. Magnetics, IEEE Transactions on 2001; 37(1):135-138.

34. Matthies HG, Niekamp R, Steindorf J. Algorithms for strong coupling procedures. Computer Methods in Applied Mechanics and Engineering 2006; 195(17):2028-2049.

35. Farajpour I, Atamturktur S. Optimization-based strong coupling procedure for partitioned analysis. Journal of Computing in Civil Engineering 2012; 26(5):648-660.

36. Leiva JS, Blanco PJ, Buscaglia GC. Iterative strong coupling of dimensionally heterogeneous models. International journal for numerical methods in engineering 2010; 81(12):1558-1580.

37. Dettmer WG, Perić D. A new staggered scheme for fluid-structure interaction. International Journal for Numerical Methods in Engineering 2013; 93(1):1-22.

38. Lee WS, Kwon KC, Kim BK, Cho JH, Young S. Frequency-shifting analysis of electrostatically tunable micromechanical actuator. Journal of Modeling and Simulation of Micro-systems 2001; 2(1):83-88.

39. Leonard P, Rodger D. Some aspects of two-and three-dimensional transient-eddy-current modelling using finite elements and single-step time-marching algorithms. IEE Proceedings A (Physical Science, Measurement and Instrumentation, Management and Education, Reviews) 1988; 135(3):159-166.

40. Zienkiewicz O, Wood W, Hine N, Taylor R. A unified set of single step algorithms. part 1: General formulation and applications. International Journal for Numerical Methods in Engineering 1984; 20(8):1529-1552.

41. Richard L, Burden J. Douglas faires, numerical analysis 1988.

42. Ansys 2014. URL http://www. ansys.com.

43. Adina 2014. URL http: //www. adina.com.

44. Ida N. Team problem 9 velocity effects and low level fields in axisymmetric geometries. Proceedings of the Vancouver TEAM WORKSHOP at the University of British Columbia, 1988.

45. Freeman E, Lowther D. Team workshop problem 17 the jumping ring.

46. Karl H, Fetzer J, Kurz S, Lehner G, Rucker WM. Description of team workshop problem 28: an electrodynamic levitation device. Proceedings of the TEAM Workshop in the Sixth Round, Citeseer, 1997; 48-51.

47. Msc adams 2012. URL http://www. mscsoftware.com/product/adams.

48. Impinna F, Detoni J, Amati N, Tonoli A. Passive magnetic levitation of rotors on axial electrodynamic bearings. Journal of Sound and Vibration 2012;

49. Tonoli A, Amati N, Impinna F, Detoni JG, Bleuler H, Sandtner J. Dynamic modeling and experimental validation of axial electrodynamic bearings. Proceedings of the 12th International Symposium on Magnetic Bearings, 2011; 68.

50. Amati N, Tonoli A, Impinna F, Girardello Detoni J. A solution for the stabilization of electrodynamic bearings: Modeling and experimental validation. Journal of Vibration and Acoustics 2011; 133:021 004-1.

51. Diez-Jimenez E, Perez-Diaz JL, Garcia-Prada JC. Local model for magnet-superconductor mechanical interaction: Experimental verification. Journal of Applied Physics 2011; 109(6):063 901-063901.

52. Valiente-Blanco I, Diez-Jimenez E, Perez-Diaz JL. Alignment effect between a magnet over a superconductor cylinder in the meissner state. Journal Of Applied Physics 2011; 109(7):07E704-07E704.

53. Albunni MN, Rischmuller V, Fritzsche T, Lohmann B. Model-order reduction of moving nonlinear electromagnetic devices. Magnetics, IEEE Transactions on 2008; 44(7):1822-1829.

54. Shabana AA. Computational dynamics. John Wiley \& Sons, 2009. 
55. Musolino A, Rizzo R. Numerical analysis of brush commutation in helical coil electromagnetic launchers. Science, Measurement \& Technology, IET 2011; 5(4):147-154.

56. Zhang Q, Hisada T. Studies of the strong coupling and weak coupling methods in fsi analysis. International journal for numerical methods in engineering 2004; 60(12):2013-2029. 